\title{
An updated survey of globular clusters in M 31
}

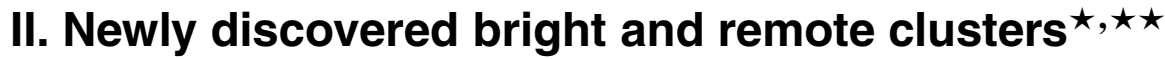

\author{
S. Galleti ${ }^{1,2}$, M. Bellazzini ${ }^{2}$, L. Federici ${ }^{2}$, A. Buzzoni ${ }^{2}$, and F. Fusi Pecci ${ }^{2}$ \\ 1 Università di Bologna, Dipartimento di Astronomia Via Ranzani 1, 40127 Bologna, Italy \\ e-mail: silvia.galleti2@unibo.it \\ 2 INAF - Osservatorio Astronomico di Bologna, Via Ranzani 1, 40127 Bologna, Italy \\ e-mail: [michele.bellazzini;luciana.federici;alberto.buzzoni;flavio.fusipecci]@oabo.inaf.it \\ Received 4 May 2007 / Accepted 22 May 2007
}

ABSTRACT

\begin{abstract}
Aims. We present the first results of a large spectroscopic survey of candidate globular clusters located in the extreme outskirts of the nearby M 31 galaxy. The survey is aimed at ascertaining the nature of the selected candidates to increase the sample of confirmed M 31 clusters lying more that $2^{\circ}$ away from the center of the galaxy.

Methods. We obtained low resolution spectra $(\lambda / \Delta \lambda \simeq 800-1300)$ of 48 targets selected from the Extended Source Catalogue of 2MASS, as in Galleti et al. (2005, A\&A, 436, 535). The observed candidates have been robustly classified according to their radial velocity and by verifying their extended/point-source nature from ground-based optical images. We have also obtained a spectrum and a radial velocity estimate for the remote M 31 globular discovered by Martin et al. (2006b, MNRAS, 371, 1983).

Results. Among the 48 observed candidates clusters we found: 35 background galaxies, 8 foreground Galactic stars, and 5 genuine remote globular clusters. One of them has been already identified independently by Mackey et al. (2007, ApJ, 655, L85), their GC1; the other four are completely new discoveries: B516, B517, B518, B519. The newly discovered clusters lie at projected distance $40 \mathrm{kpc}$ $\lesssim R_{\mathrm{p}} \lesssim 100 \mathrm{kpc}$ from the center of M 31, and have absolute integrated magnitude $-9.5 \lesssim M_{V} \lesssim-7.5$. For all the observed clusters we have measured the strongest Lick indices and we have obtained spectroscopic metallicity estimates. Mackey-GC1, Martin-GC1, B517 and B518 have spectra typical of old and metal poor globular clusters ([Fe/H] $\lesssim-1.3)$; B519 appears old but quite metal-rich $([\mathrm{Fe} / \mathrm{H}] \simeq-0.5) ; \mathrm{B} 516$ presents very strong Balmer absorption lines: if this is indeed a cluster it should have a relatively young age (likely $<2$ Gyr).

Conclusions. The present analysis nearly doubles the number of M 31 globulars at $R_{\mathrm{p}} \geq 40 \mathrm{kpc}$. At odds with the Milky Way, M 31 appears to have a significant population of very bright globular clusters in its extreme outskirts.
\end{abstract}

Key words. galaxies: individual: M 31 - galaxies: star clusters - catalogs — galaxies: Local Group

\section{Introduction}

The study of globular clusters (GC) systems is a fundamental astrophysical tool to investigate the formation and the evolution of distant galaxies. GCs are ubiquitous and abundant in virtually any type of galaxy; they are intrinsically bright and can be identified even at large distances; their integrated colors and spectra are relatively easy to interpret, since they typically host a Simple Stellar Population (Renzini \& Fusi Pecci 1988); their kinematics are powerful probes for the gravitational potential of their parent galaxy (see Brodie \& Strader 2006, and references therein).

In this context, the GC system of our next neighbor spiral galaxy, M 31, plays a key and twofold role:

- It is the richest GC system that we can study with the same integrated-light methods that we apply to any distant galaxy, and whose individual GCs can also be resolved into stars

* Appendices A and B are only available in electronic form at http://www . aanda.org

$\star \star$ Based on observations made with the Italian Telescopio Nazionale Galileo (TNG) operated on the island of La Palma by the Fundación Galileo Galilei of the INAF (Istituto Nazionale di Astrofisica) at the Spanish Observatorio del Roque de los Muchachos of the Instituto de Astrofisica de Canarias. Based on observations made with the Cassini Telescope (Loiano, Italy). The Cassini telescope is operated by INAF Osservatorio Astronomico di Bologna. with HST. This provides a fundamental sanity check for our observations of distant GC systems.

- It has a much larger number of members ( $475 \pm 25$, as estimated by Barmby et al. (2001) with respect to the GC system of the Milky Way ( $\simeq 150$ GCs), and, apparently, a larger variety of cluster "species" (including, for example, the massive young disc clusters (BLCC) described by Fusi Pecci et al. (2005), or the Extended Clusters (EC) discovered by Huxor et al. 2005). This provides the opportunity to study in detail systems that are rare or have no counterpart in our own Galaxy.

In spite of uninterrupted study since the times of Hubble (1932), we are still lacking a complete knowledge of the GC system of M 31 (Barmby et al. 2001; Galleti et al. 2006a, hereafter G06a). At present, we know more than 350 confirmed members, but hundreds of candidates are still to be checked (G06a) and any new survey finds out new clusters or promising candidates (see, for example, Mochejska et al. 1998; Huxor et al. 2004, hereafter H04; G06a and references therein). In particular, some recent studies (H04; Galleti et al. 2005, hereafter G05; Martin et al. 2006b, hereafter M06b) have opened a window on a realm that was completely unexplored: that of remote M 31 clusters, i.e. those lying at a projected distance $\left(R_{\mathrm{p}}\right)$ larger than $30-40 \mathrm{kpc}$ from the center of the galaxy. 
Until two years ago, the outermost M 31 cluster known was G1, located at $R_{\mathrm{p}} \simeq 35 \mathrm{kpc}$. This situation was quite disappointing, since in the Milky Way there are seven clusters lying at $R_{\mathrm{GC}}>40 \mathrm{kpc}$, while M 31 - which, as said, has a much richer GC system - seemed to have none. In G05 we presented a method to select candidate remote GCs in M 31 from the Extended Sources Catalogue of 2MASS (Skrutskie et al. 2006). The nature of the selected candidates must be subsequently ascertained by means of low resolution spectra that provide the radial velocity estimate by which a genuine globular cluster can be told from background galaxies or foreground stars (see G06a and references therein). In G05 we also presented the spectroscopic follow up of two of our candidates that lead to the discovery of

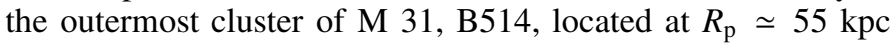
from the center of M 31. The observed spectra indicated old age and low metallicity for B514. Subsequent follow up with HST ACS/WFC allowed us to obtain a deep Color Magnitude Diagram (CMD), confirming B514 as a genuine old and metalpoor, bright GC $\left(M_{V} \simeq-9.1\right)$, with a blue Horizontal Branch (Galleti et al. 2006b, hereafter G06b). Later, M06b identified an even more extreme cluster at $R_{\mathrm{p}} \sim 118 \mathrm{kpc}$; very recently, Mackey et al. (2007) presented ACS/WFC photometry of ten additional clusters from $\mathrm{H} 04$, three of them lying at $R_{\mathrm{p}}>40 \mathrm{kpc}^{1}$.

Here we present the results of the first year of our ongoing survey for remote M 31 globular clusters whose earliest results were described in $\mathrm{G}_{0} 5^{2}$; we report on the discovery of four new bright globular clusters located at projected distance $40 \mathrm{kpc} \lesssim$ $R_{\mathrm{p}} \lesssim 100 \mathrm{kpc}$ from the center of M 31 .

All over the paper we will adopt $D=783 \mathrm{kpc}$ for M 31, from McConnachie et al. (2005); at this distance, $1^{\circ}$ corresponds to $\simeq 13.7 \mathrm{kpc}, 1^{\prime}$ to $\simeq 228 \mathrm{pc}$. $V_{\mathrm{r}}=-301 \mathrm{~km} \mathrm{~s}^{-1}$ is adopted as the systemic radial velocity of M 31 , as in G06a.

\subsection{Selection of candidate remote clusters}

The catalogues from the 2MASS all-sky survey (Skrutskie et al. 2006) provide the possibility of selecting interesting sources in arbitrarily large areas of the sky around M 31. In Galleti et al. (2004) - hereafter G04 - we searched for the counterparts of known M 31 GCs and candidates GCs (CGCs) in 2MASS and we found that the large majority $(92 \%)$ of genuine GCs are included in the Point Source Catalogue (PSC), while only the remaining $8 \%$ is in the Extended Source Catalogue (XSC). This statistic suggests that if a candidate M 31 GC is included in the $\mathrm{XSC}$ it is very likely a genuine extended source; in principle, the selection of candidates from the XSC should overcome any contamination from foreground stars. Therefore, a CGC selected from the XSC can only be a genuine GC or a distant galaxy in the background: a simple low resolution spectrum is sufficient to tell one case from the other since M 31 GCs have radial velocities around $V_{\mathrm{r}}=-301 \pm 480 \mathrm{~km} \mathrm{~s}^{-1}$, while background galaxies have cosmological recession velocities $\left(V_{\mathrm{r}}>+5000 \mathrm{~km} \mathrm{~s}^{-1}\right.$; G06a).

Actual selection criteria have evolved while the survey was ongoing and will presumably be further refined and adjusted in the future. Here we describe the preliminary selection procedure that has been adopted until now.

First of all, we considered sources extracted from 2MASSXSC located within a $\sim 20^{\circ} \times 20^{\circ}$ area around M 31 excluding

\footnotetext{
1 Plus B514 that they re-observed, and that is named GC4 in their list.

2 In parallel we are carrying on a survey to ascertain the nature of already known CGCs, drawn from the Revised Bologna Catalogue (G06a), see http://WwW . bo . astro.it/M31
}

the innermost $2^{\circ}$. We limited the extraction to well-behaved sources that have valid measures of the magnitude in $J, H$, and $K$ (13006 sources). To reduce the number of eligible sources to a more manageable number we impose three strong selection criteria, tailored on the observed characteristic of confirmed M 31 GCs:

1. GCs are quite round in shape, in general. M 31 appear to host clusters of larger ellipticity with respect to the Milky Way, still there is no known GC having $e=1 .-b / a>0.4$ (Barmby et al. 2002). Since the XSC provides the axis ratio $(b / a)$ for all the listed sources we can impose a limit in $e$ to the selected sources. To exclude from the sample all the disk galaxies seen with a significant inclination and very elongated elliptical galaxies we retained only sources having $e \leq 0.4$.

2. A very broad limit in non reddening-corrected color $(J-K \leq$ 1.2) was adopted to exclude the reddest early type galaxies, that are the most abundant contaminants in the sample.

3 . The characteristic size (half-light-radius $r_{\mathrm{h}}$ ) of globular clusters at the distance of M 31 is $0.3^{\prime \prime} \underset{<}{\sim} r_{\mathrm{h}} \underset{<}{\sim} 9^{\prime \prime}$ (Barmby et al. 2002). To reject relatively nearby background galaxies that may have large projected sizes we excluded all the sources having characteristic radius measured in the $J$ images, $R_{J}>10^{\prime \prime}$. We chose $R_{J}$ because $J$ images have the highest $\mathrm{S} / \mathrm{N}$ in $2 \mathrm{MASS}$, therefore the most reliable measure of the size of an object is obtained in this passband.

Using the above criteria, we selected $\sim 1800$ objects that were finally submitted to direct visual inspection on DSS2 images ${ }^{3}$. In this phase we mainly rejected nearly-face-on spirals and/or irregular galaxies that were not pruned by automatic criteria. The visual analysis of possible candidates is currently ongoing. At present we have inspected the images of the first 280 of them (randomly chosen): 109 passed the "visual inspection test" and were retained in the final list of good candidates that deserve spectroscopic follow-up. Here we describe the followup of 48 candidates. If we include the results of the pilot project (G05), at the present stage of advancement of the survey we have obtained spectra of 50 candidates and we obtained a harvest of six bona-fide remote M 31 GCs. Two of them were independently discovered also by H04 (see also Mackey et al. 2007), four are completely new discoveries. The global success rate of our selection procedure is $12 \%$.

\subsubsection{Selection biases}

From the above description of our selection criteria it is quite clear that our final sample is subject to several biases. By definition we cannot find very elliptical, very red and/or very extended clusters (as those identified by Huxor et al. 2005). As we draw our candidates from the XSC, we cannot select any cluster appearing as point-like in 2MASS (G04). A limit in integrated luminosity is automatically imposed by the limiting magnitude of $2 \mathrm{MASS}^{4}$; moreover we tend to favor brighter candidates when we perform the follow-up observations, for obvious reasons of

\footnotetext{
3 The DSS2 images were obtained from the site http: //archive.eso.org/dss/dss

4 The limiting magnitude of 2MASS data roughly corresponds to $M_{V} \sim-6.5$ in the Luminosity Function of M 31 GCs. Note also that we always used standard 2MASS data, not the recently released "6x" data (http://www.ipac.caltech.edu/2mass/releases/allsky/ doc/seca3_1.html) that are $\sim 1$ mag deeper, but are limited to a central field of area $2.8 \mathrm{deg}^{2}$.
} 
convenience. We clearly caution the reader that the goal of the present survey is to find out as many remote M 31 GCs as possible, but the survey cannot be by no means complete or fully objective. In spite of this, the results demonstrate that it is quite useful in finding out members of a population of M 31 clusters that has remained completely hidden until a couple of years ago.

H04 and M06b identified their remote M 31 GCs from wide and deep optical surveys (INT-WFC and MEGACAM surveys, see Ibata et al. 2001, 2004 and M06b). They can certainly reach fainter clusters and in many cases GCs are partially resolved in their images (H04), hence quite easy to identify even without spectroscopic follow-up (in fact, they are taking into consideration only objects that are - at least partially - resolved into stars). On the other hand, 2MASS allows us to survey a much wider area of the sky, including large portions of the outskirts of M 31 that are not reached by the INT-WFC and MEGACAM surveys. In this sense it is interesting to note that three of the four newly discovered clusters presented here lie in regions of the sky not covered by these optical surveys, while one is outside of the INT-WFC area but is included into the MEGACAM survey (see Sect. 3, below).

\section{Observations and data reduction}

A database of spectra for 48 targets has been assembled through several observing runs, using different instruments (see below). The main aim of the survey is to obtain low resolution spectra of candidate M 31 clusters to measure their radial velocity $\left(\mathrm{RV} ; V_{\mathrm{r}}\right)$, that allows us to ascertain if the considered candidate is a genuine GCs or a background galaxy, according to the criteria discussed in G06a. In some cases we had also to verify the extended nature of the candidates with supplementary imaging, using the technique described in G06a (see below).

\subsection{DOLORES data}

The imager/spectrograph DoLoRes at the $3.52 \mathrm{~m}$ TNG telescope in La Palma (Canary Island, Spain) was used in service mode in various nights during the period October - December, 2005 (Run 1), and in visitor mode in the nights of October 10-15, 2006 (Run 2), to acquire long slit spectra of 38 M 31 CGCs. DoLoRes is equipped with a $2048 \times 2048 \mathrm{px}^{2}$ thinned and backilluminated Loral CCD array with a total field of view of $9.4^{\prime} \times$ 9.4'. The adopted MG-B grism yields a resolution of $6 \AA(R=$ 875) with $1^{\prime \prime}$ slit, and covers the spectral range $3800 \AA<\lambda<$ $6800 \AA$. Our typical exposure times range from 15 to $20 \mathrm{~min}$ for Run 1 and from 45 to 60 min for Run 2, giving spectra with typical $S / N \sim 35$ per resolution element. In both runs, high $\mathrm{S} / \mathrm{N}$ spectra of the bright clusters B158 and B225 were also obtained, to be used as templates for the estimates of $V_{\mathrm{r}}$ as in G05 and G06a.

During Run 1, technical problems prevented the acquisition of a reference lamp after each spectrum. For this reason the wavelength calibration of science spectra was of low quality and the typical uncertainty in radial velocity was $\sim \pm 200 \mathrm{~km} \mathrm{~s}^{-1}$. This allowed a good discrimination between GCs and background galaxies but the $V_{\mathrm{r}}$ estimates of GCs were completely unusable for any other purpose. The GCs identified during this run have been re-observed later at the Cassini telescope (see below).

A He lamp spectrum was acquired after each science frame for wavelength calibration during Run 2. Spectra of the adopted radial velocity template clusters have been obtained during each observing night, to ensure the self consistency of the $V_{\mathrm{r}}$ scale over the whole run. Bias subtraction, flat-fielding, and sky subtraction were performed using standard packages in IRAF, as described in G05.

\subsection{BFOSC data}

Long slit spectra for 16 CGCs in M 31 were obtained with the low resolution spectrograph BFOSC (Gualandi \& Merighi 2001) mounted at the $1.52 \mathrm{~m}$ Cassini Telescope of the Loiano Observatory, near Bologna (Italy), during several runs in 2006: August 19-22 (Run 1), September 1-2 (Run 2), October 2527 (Run 3), and November 22-23 (Run 4). In six cases we reobserved interesting candidates identified during TNG-Run 1 ; in two cases we observed a given target in two different runs to obtain a spectrum of sufficient $\mathrm{S} / \mathrm{N}$ for subsequent analysis. During Run 1 we acquired a spectrum of the remote cluster recently discovered by M06b; in the following we will refer to this clusters as to Martin-GC1.

Nearly $60 \%$ of the nights were suitable for useful observations. The typical seeing was $1.5^{\prime \prime}-2.5^{\prime \prime}$ FWHM. The detector was a thinned, back illuminated EEV CCD, with $1300 \times$ $1340 \mathrm{px}^{2}$. A $1.5^{\prime \prime}$ slit was used. The adopted set-up provides a spectral resolution $\Delta \lambda=4.1 \AA(\lambda / \Delta \lambda \sim 1300)$ and covers the range $4200 \AA<\lambda<6600 \AA$. We took a He-Ar calibration lamp spectrum after each scientific exposure, maintaining the same pointing of the telescope. Integrations were typically 60 min per exposure, yielding spectra with characteristic signalto-noise ratio $S / N \sim 24$ per resolution element. During each observing night we also observed, with the same set-up, at least a couple of template targets that we adopted as radial velocity standards. Such targets are extracted from the list of bright M 31 clusters (Master RV templates) for which we have constructed very high $\mathrm{S} / \mathrm{N}$ template spectra by stacking several spectra obtained in several BFOSC observing runs (see Appendix A for details of the procedure published only in the electronic edition of the Journal). In practice the spectra of RV standard that are obtained during each observing night are used to ensure that the measures obtained during the night are in the RV scale defined by the Master RV templates. The data reduction steps were performed using standard packages in IRAF, as for the Dolores spectra, above.

\subsection{Radial velocities}

The heliocentric radial velocities $\left(V_{\mathrm{r}}\right)$ of the candidate globular clusters were obtained by cross-correlation (CC) with the template spectra, using the IRAF/fxcor package (see Tonry \& Davis 1979 for details of the technique). We applied a square filter to dampen the highest and lowest frequency Fourier components; if unfiltered, these frequencies produced broad features that masked the narrow CC peaks. We fit the CC peaks with Gaussians. The typical internal velocity errors on a single measure were $\sim 50 \mathrm{~km} \mathrm{~s}^{-1}$ for BFOSC and $\sim 65 \mathrm{~km} \mathrm{~s}^{-1}$ for DoLoRes spectra.

In both cases we averaged the RV measures obtained by the $\mathrm{CC}$ with different template spectra and we adopted the standard deviation of the different estimates as our final error on RV, as done in G06a. This procedure allows a significant reduction of the uncertainty on $\mathrm{RV}$, in particular for the BFOSC spectra, that were cross-correlated with five very-high-S/N spectra from our Master RV Templates set (see Appendix A, in online edition). Note that all our CC RV estimates have been independently checked by measuring the average red/blue-shift on the strongest 
Table 1. Observed candidates globular cluster.

\begin{tabular}{|c|c|c|c|c|c|c|c|c|c|c|}
\hline 2Mass-Name & $\overline{\bar{K}}$ & $\overline{\overline{V_{\mathrm{r}}}}$ & $\pm \epsilon_{V \mathrm{r}}$ & Source & $V_{\mathrm{r}}$ & $\pm \epsilon_{V \mathrm{r}}$ & Source & $\bar{R}$ & $\overline{\mathrm{E} / \mathrm{PS}}$ & Name \\
\hline 2MASX-J00255466+4057060 & 12.37 & $12200\left(\mathrm{EL}^{a}\right)$ & 500 & R1 TNG & & & & & & \\
\hline 2MASX-J00264769+3944463 & 13.64 & $-350^{1}$ & 200 & R1 TNG & -219 & 15 & R1 LOI & 1.27 & $\mathrm{E}$ & Mackey-GC1 \\
\hline 2MASX-J00285169+3905167 & 13.82 & $-100^{1}$ & 200 & R1 TNG & 22 & 14 & R1 LOI & 1.01 & PS & \\
\hline 2MASX-J00294581+3729300 & 11.97 & $10800^{1}$ & 1200 & R1 TNG & 11421 & 12 & R2 LOI & & & \\
\hline 2MASX-J00301921+4117203 & 14.25 & M Star( $\left(^{b}\right)$ & 200 & R1 TNG & & & & & & \\
\hline 2MASX-J00305689+4520599 & 13.82 & 19400 & 1500 & R1 TNG & & & & & & \\
\hline 2MASX-J00313051+4033153 & 14.15 & M Star & 38 & R2 TNG & & & & & & \\
\hline 2MASX-J00313771+4034163 & 14.00 & 52861 & 121 & R2 TNG & & & & & & \\
\hline 2MASX-J00350474+4235316 & 13.61 & $19700^{1}$ & 1200 & R1 TNG & 19638 & 43 & R2 LOI & & & \\
\hline 2MASX-J00442168+3843319 & 14.34 & $-150^{1}$ & 200 & R1 TNG & -23 & 14 & R1 LOI & 0.99 & PS & \\
\hline 2MASX-J00491125+3806514 & 12.58 & $11400(\mathrm{EL})$ & 500 & R1 TNG & & & & & & \\
\hline 2MASX-J00492851+3826166 & 14.22 & M Star & 200 & R1 TNG & & & & & & \\
\hline 2MASX-J00553861+4524413 & 13.77 & $-350^{1}$ & 200 & R1 TNG & -181 & 5 & R1 LOI & 1.09 & $\mathrm{E}$ & B516 \\
\hline 2MASX-J01011110+3915047 & 13.44 & 18100 & 1500 & R1 TNG & & & & & & \\
\hline 2MASX-J01014341+4024207 & 13.53 & 24900 & 500 & R1 TNG & & & & & & \\
\hline 2MASX-J00272474+4507360 & 14.23 & M Star & 38 & R2 TNG & & & & & & \\
\hline 2MASX-J00312909+4053553 & 13.22 & 20765 & 22 & R1 LOI & & & & & & \\
\hline 2MASX-J00382837+4608217 & 13.48 & -22 & 21 & R1 LOI & -18 & 9 & R2 LOI & 1.00 & PS & \\
\hline 2MASX-J00290822+3928357 & 12.41 & $10887(\mathrm{EL})$ & 18 & R1 LOI & & & & & & \\
\hline 2MASX-J00291911+3825166 & 12.98 & 5830 & 15 & R1 LOI & & & & & & \\
\hline 2MASX-J00240694+4459072 & 14.24 & $45340^{2}$ & 24 & R1 LOI & & & R2 LOI & & & \\
\hline 2MASX-J00515568+4707229 & 13.53 & 14491 & 16 & R2 LOI & & & & & & \\
\hline 2MASX-J00271402+3548121 & 13.17 & 18667 & 24 & R2 LOI & & & & & & \\
\hline 2MASX-J00422528+3742110 & 13.58 & 11520 & 57 & R3 LOI & & & & & & \\
\hline 2MASX-J00595989+4154068 & 14.16 & -272 & 54 & R2 TNG & & & & 1.33 & $\mathrm{E}$ & B517 \\
\hline 2MASX-J01002390+4225505 & 14.02 & 31228 & 45 & R2 TNG & & & & & & \\
\hline 2MASX-J01003776+4213045 & 14.00 & 32105 & 84 & R2 TNG & & & & & & \\
\hline 2MASX-J00252922+3750258 & 14.34 & 29579 & 73 & R2 TNG & & & & & & \\
\hline 2MASX-J00273099+4008015 & 14.18 & 21384 & 71 & R2 TNG & & & & & & \\
\hline 2MASX-J00294944+4143240 & 14.07 & M Star & 24 & R2 TNG & & & & & & \\
\hline 2MASX-J00364114+3734534 & 14.43 & 46257 & 21 & R2 TNG & & & & & & \\
\hline 2MASX-J00370125+3738403 & 14.47 & 54659 & 31 & R2 TNG & & & & & & \\
\hline 2MASX-J00373023+3626149 & 13.04 & 15875 & 45 & R2 TNG & & & & & & \\
\hline 2MASX-J00254284+4125357 & 13.51 & 44726 & 85 & R2 TNG & & & & & & \\
\hline 2MASX-J00262799+4416097 & 14.18 & 48977 & 111 & R2 TNG & & & & & & \\
\hline 2MASX-J00355252+3751415 & 14.06 & 28412 & 68 & R2 TNG & & & & & & \\
\hline 2MASX-J00423531+3731294 & 13.98 & 38784 & 72 & R2 TNG & & & & & & \\
\hline 2MASX-J00425347+3909135 & 14.21 & 52861 & 121 & R2 TNG & & & & & & \\
\hline 2MASX-J00543871+4507287 & 13.88 & 25418 & 54 & R2 TNG & & & & & & \\
\hline 2MASX-J00565026+4008522 & 14.07 & 28742 & 68 & R2 TNG & & & & & & \\
\hline 2MASX-J01035016+4320352 & 14.31 & 63234 & 34 & R2 TNG & & & & & & \\
\hline 2MASX-J00145479+3905041 & 14.40 & -200 & 48 & R2 TNG & & & & 1.06 & $\mathrm{E}$ & B518 \\
\hline 2MASX-J00162141+3412002 & 14.05 & 51033 & 154 & R2 TNG & & & & & & \\
\hline 2MASX-J00213516+4813322 & 14.02 & 24782 & 24 & R2 TNG & & & & & & \\
\hline 2MASX-J00513014+3407389 & 13.99 & -268 & 47 & R2 TNG & & & & 1.36 & $\mathrm{E}$ & B519 \\
\hline 2MASX-J01100019+4024210 & 13.77 & 17236 & 58 & R2 TNG & & & & & & \\
\hline 2MASX-J00020201+5136242 & 13.86 & 24441 & 13 & R4 LOI & & & & & & \\
\hline 2MASX-J00061191+4130134 & 13.17 & 19054 & 7 & R4 LOI & & & & & & \\
\hline 2MASS-J00504245+3254587 & 13.37 & -312 & 17 & R1 LOI & & & & & & Martin-GC1 \\
\hline
\end{tabular}

${ }^{a}$ Object with prominent emission lines in its spectrum. ${ }^{b}$ Object with M type spectrum (prominent TiO bands).

R1, R2, R3 etc., refer to the various observing runs at the TNG and Cassini (Loiano) telescopes, see Sect. 2. The 2MASS counterpart of the remote cluster discovered by Martin et al. (2006b), here named Martin-GC1, is in 2MASS-PSC instead of 2MASS-XSC as all other entries. Mackey-GC1 is GC1 from Mackey et al. (2007).

${ }^{1}$ Very uncertain RV estimates obtained during Run 1 at TNG; superseded by the estimates from other sources.

${ }^{2}$ The radial velocity has been estimated from the stacked spectrum obtained by co-adding the spectra acquired in Run 1 and Run 2 at the Cassini telescope.

lines identified in each spectrum, using standard IRAF/rv tasks. The RVs obtained with the two independent methods are fully consistent, within the observing errors.

\subsection{Optical imaging}

To gain insight on the morphology of the most promising candidates and, in particular, to discriminate between truly Extended sources and actual stars (Point Sources) we obtained BFOSC optical images in white light for eight of the CGCs listed in Table 1 (those having the R (9) and E/PS (10) columns filled), as done in G06a. The observations were performed during Run 2, 3 and 4.

In imaging mode, BFOSC has a pixel scale of $0.58^{\prime \prime} / \mathrm{px}$ and a total field of view of $13.0^{\prime} \times 12.6^{\prime}$. The exposure times ranged between 1 and $5 \mathrm{~min}$, depending on the brightness of the target and 


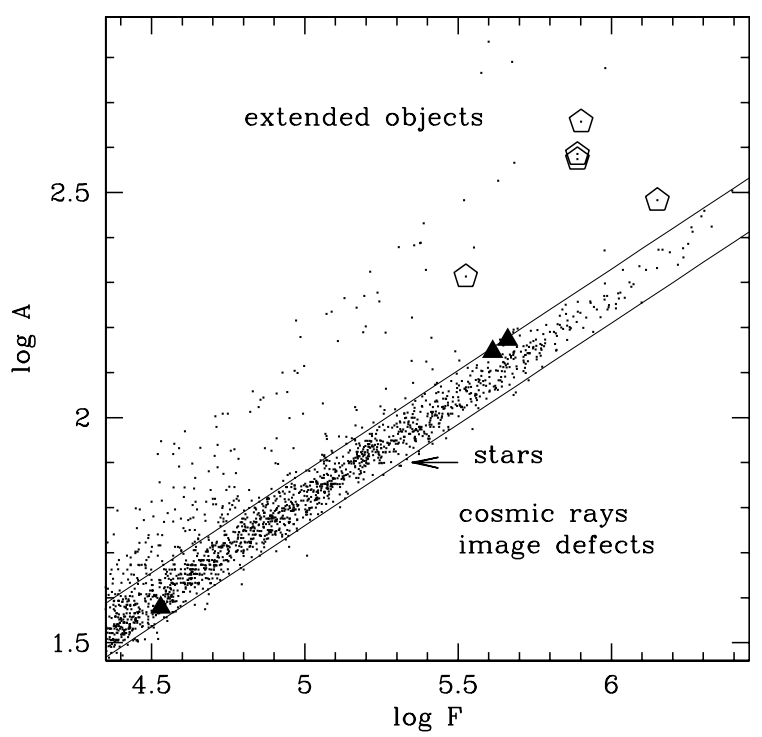

Fig. 1. $F-A$ diagrams from optical imaging for the considered targets (see G06a for discussion of the method). The pentagons are the CGCs classified as Extended objects (B516, B517, B518, B519 and Mackey-GC1), filled triangles are the CGCs classified as Point Sources, i.e. 2MASX-J00285169+3905167, 2MASX-J00442168+3843319, and 2MASX-J00382837+4608217. The parallel lines enclose the strip of the diagram that is populated by bona-fide stars (PS). The imaging of different fields have been renormalized to a unique scale by matching the sequence of stars.

on the atmospheric conditions. The nights were not photometric but clear; the typical seeing was around $1.5^{\prime \prime}-2^{\prime \prime}$ FWHM.

The images have been corrected for bias and flat field with standard IRAF procedures. Relative photometry, FWHM and morphological parameters of each source in the frame - down to a $5 \sigma$ threshold - was obtained with Sextractor (Bertin \& Arnouts 1996). Only non-saturated and isolated sources are retained in the final catalogs (Sextractor quality flag $=0$ ). The relatively wide field of view of BFOSC allows the simultaneous imaging of the target and several field stars, thus generally allowing a robust discrimination between Extended and Point Sources (see G06a and below).

\section{Classification of candidates GC}

The essential results of the survey are reported in Table 1, that lists the 2MASS designation of the surveyed CGCs (name; Col. 1), the $K$ magnitude from 2MASS (transformed as in G04; Col. 2), a first estimate of RV, the associated uncertainties and the observing run during which the spectra were acquired (Cols. 3, 4 , and 5 , respectively), a second - typically more accurate RV estimate, the associated uncertainty and the observing run (Cols. 6, 7, and 8, respectively), the ratio $R$ between the $F W H M$ of the target and the $F W H M$ of stars as measured on optical images (Col. 9), a morphological classification based on the Flux-Area diagram $(\mathrm{E}=$ Extended, $\mathrm{PS}=$ Point Source, Col. 10; see G06a and below), and the name assigned here or by other authors to the candidates that have been confirmed as bonafide GCs (Col. 11). The RV estimates of column 6 supersedes those presented in Col. 3, when both are present (see above). Note that the 2MASS designation provides also the J2000 equatorial coordinates of the object: for example the coordinates of 2MASX-J00255466+4057060 are RA $=00^{\mathrm{h}} 25^{\mathrm{m}} 54.7^{\mathrm{s}}$ and Dec $=40^{\circ} 57^{\prime} 06.0^{\prime \prime}$.
Most of the classification work is quite straightforward: Table 1 contains 35 sources that have large recession velocity $\left(V_{\mathrm{r}}>+5000 \mathrm{~km} \mathrm{~s}^{-1}\right)$, clearly of cosmologic origin. Hence they are distant background galaxies (class 4 in the Revised Bologna Catalogue - RBC - see G06a, and references therein ${ }^{5}$ ).

Five of the remaining 12 objects display strong TiO bands typical of M stars in their spectra. The spectra of our RV templates are clearly too different from those of these sources for a safe application of the CC technique. For this reason we don't provide RV estimates for these sources. However, since genuine Globular Clusters have spectral types earlier than K0 (Harris 1996), they clearly cannot be M 31 GCs, and we classify all of them as (likely foreground) stars (RBC class 6).

Of the remaining eight candidates, five have $V_{\mathrm{r}}<$ $-150 \mathrm{~km} \mathrm{~s}^{-1}$, hence they may be classified as genuine M 31 GCs based on their radial velocity alone, according to the criteria by G06a. The other three have RV typical of the Galactic foreground population $\left(\left\langle V_{\mathrm{r}}\right\rangle=-29 \mathrm{~km} \mathrm{~s}^{-1}\right.$ and $\sigma=42.6 \mathrm{~km} \mathrm{~s}^{-1}$, G06a) but still compatible with M 31 GCs. Their spectral type is compatible with being GCs, hence we must recur to morphological criteria to ascertain their nature. From the optical imaging described in Sect. 2.4 above we derived the ratio $R$ between the $F W H M$ of the candidate and that of stars in the same image. In general extended objects should have $R>1.0$. We find here that all the considered CGCs having $-150 \mathrm{~km} \mathrm{~s}^{-1}<V_{\mathrm{r}}<+100 \mathrm{~km} \mathrm{~s}^{-1}$ have $R \simeq 1.0$, typical of point sources. Moreover, in a Flux-Area diagram (F-A, see G06a) they lie in the characteristic strip of Point Sources (see Fig. 1). Hence we classify them as foreground stars (RBC class 6). It is somehow unexpected that our sample - selected from a catalogue of extended sources (XSC) - contains also stars (see Sect. 1.1, above). This is probably due to the modest spatial resolution of the original 2MASS images that may blend one or more stars into a spurious extended source.

All the five candidates with $V_{\mathrm{r}}<-150 \mathrm{~km} \mathrm{~s}^{-1}$ have $R \geq$ 1.06 , up to $R=1.36$, and appear as Extended sources in the $F-$ $A$ diagram of Fig. 1. Hence we classify all of them as genuine M 31 GCs (RBC class 1, but see Sect. 3.2, below). It turned out that 2MASX-J00264769+3944463 was already (independently) identified by H04; it is listed as GC1 in Mackey et al. (2007). The HST imaging by these authors confirms that this is a genuine M 31 globular. In the following we will refer to this cluster as Mackey-GC1, in analogy to the case of Martin-GC1 ${ }^{6}$, while the assigned RBC name is B520 (see below).

On the other hand, 2MASX-J00553861+4524413, 2MASXJ00595989+4154068, 2MASX-J00145479+3905041, 2MASXJ00513014+3407389, have never been recognized before as M 31 globular clusters. They are completely new discoveries and we christen them B516, B517, B518, and B5197, respectively, according to the RBC nomenclature. These new discoveries increase the number of known remote $\mathrm{M} 31$ clusters $\left(R_{\mathrm{p}} \geq 40 \mathrm{kpc}\right)$ from five (B514/Mackey-GC4, Mackey-GC1/B520, MackeyGC5, Mackey-GC10 and Martin-GC1) to nine clusters. If we consider clusters having $R_{\mathrm{p}} \geq 35 \mathrm{kpc}$ the sample increases from 8 to 12 clusters (see Fig. 8, below).

\footnotetext{
${ }^{5}$ See the Appendix B (published only in the electronic edition of the Journal).

6 These are provisional names. A. Huxor and collaborators will assess the nomenclature of the clusters they discovered in the INT-WFC and MEGACAM surveys in a paper that is in preparation (A. Huxor, private communication). In the current version of the $\mathrm{RBC}$ we have adopted the following abbreviated names for these clusters: MackeyGC1 = MCGC1, Martin-GC1 = MGC1.

7 The name B515 was assigned to a cluster recently discovered on HST images, as reported by G06a.
} 


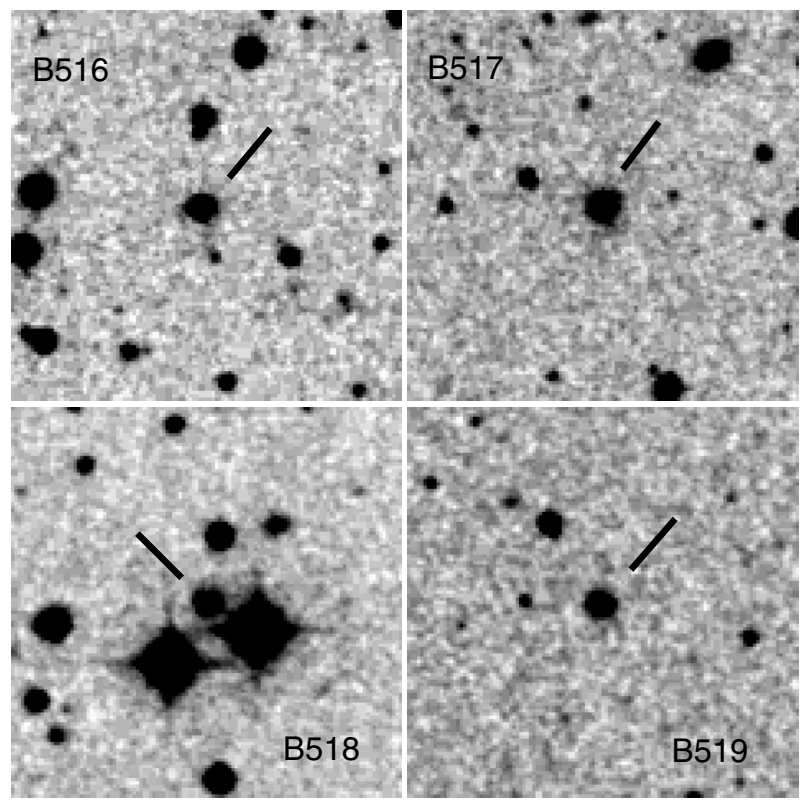

Fig. 2. DSS2 images of the newly discovered clusters. Each image is $\simeq 2^{\prime}$ on a side. North is up, East is to the left. Note that B518 lies between two very bright foreground stars.

DSS2 post stamp images of the new clusters are presented in Fig. 2. It is interesting to note that B518 and Martin-GC1 are listed in the Hyperleda database of galaxies (Paturel et al. 2003); hence they have been independently recognized as extended sources also in that catalogue. Among the targets with non-cosmological velocities, the only other source included in Hyperleda is 2MASX-J00442168+3843319 - that we clearly identify as a point source. In support to our conclusion, the NOMAD catalogue (Zacharias et al. 2004) report a total proper motion of $168 \mathrm{mas} / \mathrm{yr}$ for this source; all the targets we classified as clusters have estimated proper motions $<10 \pm 10 \mathrm{mas} / \mathrm{yr}$ (i.e. consistent with zero, if any), as expected for extra-galactic sources.

\subsection{The physical characteristics of the new clusters}

Some useful parameters of the remote clusters studied here are summarized in Table 2 . The $V$ magnitudes have been drawn from the NOMAD catalogue (Zacharias et al. 2004) and from M06b for Martin-GC1.

All the considered clusters are more than $3^{\circ}$ away from the center of M 31. With the adopted distance scale, the projected distance of these clusters goes from $R_{\mathrm{p}}=45 \mathrm{kpc}$ to $R=101 \mathrm{kpc}$. Martin-GC1 $\left(R_{\mathrm{p}}=117 \mathrm{kpc}\right)$ remains the outermost known GC of M 31, to date. Note that RGB stars belonging to the halo of M 31 have been traced out to $\sim 165 \mathrm{kpc}$ from the center of M 31 (Kalirai et al. 2006).

Near Infra Red (NIR) colors of the considered clusters ${ }^{8}$ are compared to those of the known confirmed M 31 GCs from the

\footnotetext{
${ }^{8}$ The colors of remote clusters has been corrected for extinction using the COBE-DiRBE reddening maps by Schlegel et al. 1998. The disc of M 31 is a non-subtracted source in the COBE-DiRBE maps; for this reason the reddening derived from the maps is - in general - incorrect for sources projected unto the disc itself. The remote clusters considered here are too far from the disc of M 31 to be affected from this kind of problem, hence reliable estimates of the average foreground extinction can be obtained from Schlegel et al.'s maps. For the other RBC clusters plotted in Fig. 3 we adopted $E(B-V)=0.1$, as in G06b.
}

RBC in Fig. 3. All the newly discovered clusters have colors within the range covered by known confirmed M 31 globular clusters. B516 appears very blue in $(H-K)_{0}$ and B519 appears as one of the reddest M 31 GCs. These possibly odd positions in the NIR color-color diagram may be due to (a) random fluctuations within the sizable uncertainties affecting the photometry, typically of order $\simeq 0.05-0.1 \mathrm{mag}$ for these clusters, (b) local variations of the interstellar extinction, and/or (c) intrinsic differences in physical parameters (age, metallicity).

A further insight in can be obtained by inspection of the spectra, shown in Fig. 4 (DoLoRes spectra) and Fig. 5 (BFOSC spectra). In both figures we report also the spectrum of a well known and bright M 31 GC, the metal rich cluster B225 $([\mathrm{Fe} / \mathrm{H}] \simeq-0.45 \text {, Puzia et al. 2005 })^{9}$, observed with the same instrument, for comparison.

Figure 4 shows that B517 and B518 have weaker MgI and $\mathrm{NaI}$ lines and stronger Balmer lines with respect to B225. This suggests that they may be old globulars, significantly more metal deficient than B225 (see below). On the other hand, B519 has Balmer lines very similar to B225 and metal lines as strong or stronger than B225, thus suggesting that this may be a quite metal rich globular cluster.

In Fig. 5 we plotted also the spectrum of the remote old and metal-poor cluster B514 $([\mathrm{Fe} / \mathrm{H}] \simeq-1.8, \mathrm{G} 05$, G06b). Mackey$\mathrm{GC} 1$ and Martin-GC1 have $\mathrm{MgI}$ and $\mathrm{NaI}$ lines much weaker than B225; their overall spectrum is very similar to that of B514, i.e. of an old and metal poor globular with a Blue Horizontal Branch (BHB). This is in full agreement with the results obtained by M06b and by Mackey et al. (2007) from the CMD of the clusters. On the other hand B516 shows strong NaI and weak MgI features, while Balmer's lines are much stronger that those observed in the old metal-poor and BHB clusters. These characteristics suggests a significantly younger age for this cluster.

As for B514, we measured the strongest Lick's indices that are accessible within our spectra $\left(\mathrm{Mg}_{2}, \mathrm{Mgb}, \mathrm{H}_{\beta}\right)$, following the procedure illustrated in G05 and adopting the definitions by Trager et al. (1998). The comparison between the indices measured by us and those reported by Trager et al.'s for the clusters in common (B225, B158, see Table 3, below) suggests that our indices are in reasonable agreement with the original Lick scale; however they should be considered as preliminary estimates until a more detailed comparison allows us to apply a robust transformation from our natural systems to the actual Lick system (Galleti et al. 2007, in preparation; hereafter G07). The measured indices are reported in Table 3. We use the following relation calibrated on Galactic globulars (G07), to obtain spectroscopic estimates of the metallicity from the $\mathrm{Mg}_{2}$ index

$[\mathrm{Fe} / \mathrm{H}]=-2.32+12.89 \mathrm{Mg}_{2}-15.9 \mathrm{Mg}_{2}^{2} \quad \mathrm{rms}=0.17$ dex. (1)

The resulting metallicities are reported in Table 3; they are in good agreement with those obtained from the theoretical calibration by Buzzoni et al. (1992), that are also reported in the same table, for comparison. The estimates for Mackey-GC1 and Martin-GC1 are in agreement with those presented in Mackey et al. (2007) and in M06b, respectively (i.e. $[\mathrm{Fe} / \mathrm{H}] \sim-2.0$ ). B517 and B518 seem slightly less metal deficient $([\mathrm{Fe} / \mathrm{H}] \sim$ -1.3 and $\sim-1.6$, respectively. B519 clearly emerges as a metallicity outlier among the remote clusters, having $[\mathrm{Fe} / \mathrm{H}] \simeq-0.4$. The adopted $[\mathrm{Fe} / \mathrm{H}]$ vs. $\mathrm{Mg}_{2}$ relation is valid only for classical old globular cluster. The strong Balmer lines suggest that B516

\footnotetext{
9 Puzia et al. (2005) report all metallicities in $[\mathrm{Z} / \mathrm{H}]$ dex. A transformation to $[\mathrm{Fe} / \mathrm{H}]$ has been done through the equation: $[\mathrm{Fe} / \mathrm{H}]=$ $[\mathrm{Z} / \mathrm{H}]-0.94[\alpha / \mathrm{Fe}]$ from Thomas et al. (2003).
} 
Table 2. Remote clusters: positions and magnitudes.

\begin{tabular}{lcrrrrrrr}
\hline \hline Name & $\begin{array}{r}V \\
\mathrm{mag}\end{array}$ & $\begin{array}{r}r^{\prime} \\
\mathrm{mag}^{1}\end{array}$ & $X^{\prime}$ & $Y^{\prime}$ & $R^{\prime}$ & $\begin{array}{r}R \\
\mathrm{Kpc}\end{array}$ & $\begin{array}{r}V_{\mathrm{r}} \\
\mathrm{km} \mathrm{s}^{-1}\end{array}$ & $\begin{array}{r} \pm \epsilon_{V \mathrm{r}} \\
\mathrm{km} \mathrm{s}^{-1}\end{array}$ \\
\hline Mackey-GC1 & 15.0 & 16.18 & -181.6 & 92.2 & 203.7 & 46.4 & -219 & 15 \\
B516 & 15.3 & 15.55 & 282.5 & 46.2 & 286.2 & 65.2 & -181 & 5 \\
B517 & 16.1 & 16.13 & 151.8 & -126.3 & 197.5 & 45.0 & -272 & 54 \\
B518 & 16.0 & 16.27 & -292.6 & 184.3 & 345.8 & 78.8 & -200 & 48 \\
B519 & 17.3 & - & -272.7 & -349.5 & 443.3 & 101.0 & -268 & 47 \\
Martin-GC1 & 15.5 & 15.28 & -336.4 & -338.4 & 513.9 & 117.0 & -312 & 17 \\
\hline
\end{tabular}

${ }^{1}$ Homogeneously calibrated CCD magnitudes in the Sloan Digital Sky Survey $r^{\prime}$ passband from the Carlsberg Meridian Catalogue 14 http://www . ast. cam.ac.uk/ dwe/SRF/cmc14.html.

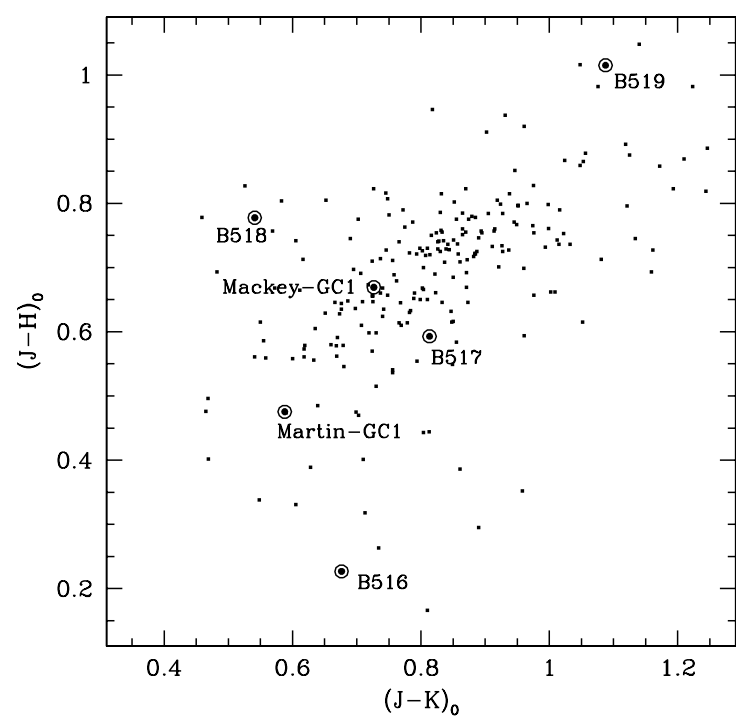

Fig. 3. De-reddened near infrared colors of confirmed M $31 \mathrm{GCs}$ from the RBC (Galleti et al. 2004; small points). A reddening of $E(B-V)=$ 0.1 has been assumed for all the clusters. The remote clusters identified in our survey are plotted as larger symbols (encircled filled circles) and labeled. Their reddening has been taken from the COBE/DIRBE maps by Schlegel et al. (1998).

may be much younger than classical globulars, hence the derived metallicity estimate is probably unreliable (consequently, it is not reported in Table 3). On the other hand, the measured $\mathrm{H}_{\beta}>4.5 \AA$ includes B516 among the Blue Luminous Compact Clusters (BLCC) as defined by Fusi Pecci et al. (2005). The integrated spectra and color of these clusters indicate ages $\leq 2 \mathrm{Gyr}$, as can be appreciated from the comparison with Buzzoni et al. (1992) models shown in Fig. 6.

\subsection{B516 and B519: supplementary investigation}

As said above, B516 and B519 fulfil all the criteria devised by G06a to classify a CGC as a genuine M 31 star cluster. However both present some peculiar property, requiring some supplementary investigation on their nature.

As said, B519 is the only one of our newly discovered clusters that lies within the boundaries of the MEGACAM survey (M06b). N.F. Martin kindly inspected the images from their deep optical survey and provided us with an image of the target and its surroundings. B519 clearly appears as an extended source, with a halo of diffuse light; the MEGACAM image fully confirms that it cannot be neither a star or a blend of two or more stars. Unfortunately, the image is not partially resolved into stars as in the case of Martin-GC1; from the mere inspection of the

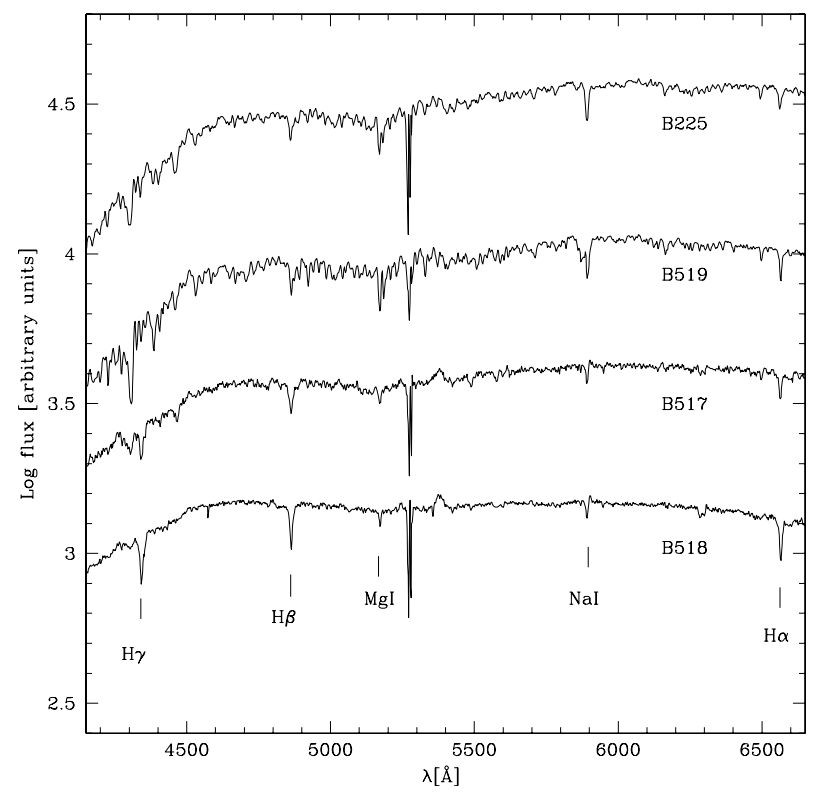

Fig. 4. DOLORES spectra of target clusters. From top to bottom, the RV template B225, a well known metal-rich cluster, reported here for comparison, B519, B517, and B518. The main spectral features have been labeled. The strong feature at $\simeq 5250 \AA$ is a spurious line due to a defect of the CCD. Note the prominent Mg lines in the spectrum of B519, suggestive of a metal content similar or larger than that of B225 $([\mathrm{Fe} / \mathrm{H}] \sim-0.45)$.

images one would have classified B519 as an elliptical galaxy. However the non-resolved nature of the image is not conclusive, since there are cases in which genuine M 31 GCs cannot be (even partially) resolved into stars even with HST imagery (see, for example, Barmby et al. 2007).

In the scenario outlined above, the final word is left to the radial velocity estimate. The estimate presented in Table 1 is obtained from a very clean, single and strong cross-correlation peak $(C C \simeq 0.4$ and Tonry \& Davis' parameter $T D R \simeq 20)$. We obtained an alternative estimate of RV from the same spectra by measuring the wavelength shift (with respect to restframe) of seven strong lines (including Balmer lines): the average velocity is $\left\langle V_{\mathrm{r}}\right\rangle=-241 \mathrm{~km} \mathrm{~s}^{-1}$, the standard deviation $\sigma=36 \mathrm{~km} \mathrm{~s}^{-1}$, in full agreement with the CC result. Since (a) it is out of doubt that B519 is a bona fide extended object, and (b) it has a velocity typical of M 31 GCs and incompatible with cosmological recession, we firmly conclude that B519 is a genuine remote - and metal rich-star cluster of $M 31$.

The RV estimate rules out a background galaxy as a viable hypothesis also for B516. Unfortunately, in this case we lack imaging of sufficient quality to rule out the possibility that it 
Table 3. Remote clusters and template clusters: lick indices and metallicities.

\begin{tabular}{lccccc}
\hline \hline Name & $\begin{array}{c}\mathrm{H}_{\beta} \\
{[\AA]}\end{array}$ & $\begin{array}{c}\mathrm{Mg}_{2} \\
{[\mathrm{mag}]}\end{array}$ & $\begin{array}{c}\mathrm{Mgb} \\
{[\AA]}\end{array}$ & $\begin{array}{c}{[\mathrm{Fe} / \mathrm{H}]_{\mathrm{Mg} 2}} \\
\mathrm{Ea} .1\end{array}$ & $\begin{array}{c}{[\mathrm{Fe} / \mathrm{H}]_{\mathrm{Mg} 2}} \\
\text { BGM92 }\end{array}$ \\
\hline Mackey-GC1 & $1.841 \pm 0.286$ & $0.023 \pm 0.007$ & $0.688 \pm 0.290$ & -2.0 & -1.9 \\
B516 & $4.818 \pm 0.146$ & $0.011 \pm 0.004$ & $0.684 \pm 0.165$ & - & - \\
B517 & $2.567 \pm 0.128$ & $0.089 \pm 0.004$ & $1.066 \pm 0.150$ & -1.3 & -1.4 \\
B518 & $3.367 \pm 0.088$ & $0.057 \pm 0.002$ & $1.000 \pm 0.106$ & -1.6 & -1.6 \\
B519 & $1.996 \pm 0.026$ & $0.195 \pm 0.001$ & $3.592 \pm 0.028$ & -0.4 & -0.6 \\
Martin-GC1 & $1.595 \pm 0.283$ & $0.020 \pm 0.007$ & $1.012 \pm 0.285$ & -2.1 & -1.9 \\
\hline B158 Loi & $1.853 \pm 0.092$ & $0.112 \pm 0.002$ & $2.390 \pm 0.090$ & -1.1 & -1.2 \\
G001 Loi & $2.239 \pm 0.103$ & $0.110 \pm 0.003$ & $2.301 \pm 0.104$ & -1.1 & -1.2 \\
B514 Loi & $2.317 \pm 0.132$ & $0.044 \pm 0.003$ & $0.422 \pm 0.137$ & -1.8 & -1.7 \\
B225 Loi & $1.725 \pm 0.043$ & $0.167 \pm 0.001$ & $3.449 \pm 0.042$ & -0.6 & -0.8 \\
B225 TNG & $1.775 \pm 0.062$ & $0.204 \pm 0.002$ & $3.163 \pm 0.068$ & -0.3 & -0.6 \\
\hline
\end{tabular}

Below the horizontal line we list the data for already known clusters, for reference.

${ }^{1}$ Metallicity from the calibration provided by Buzzoni et al. (1992), $[\mathrm{Fe} / \mathrm{H}]=7.41 \mathrm{Mg}_{2}-2.07$.

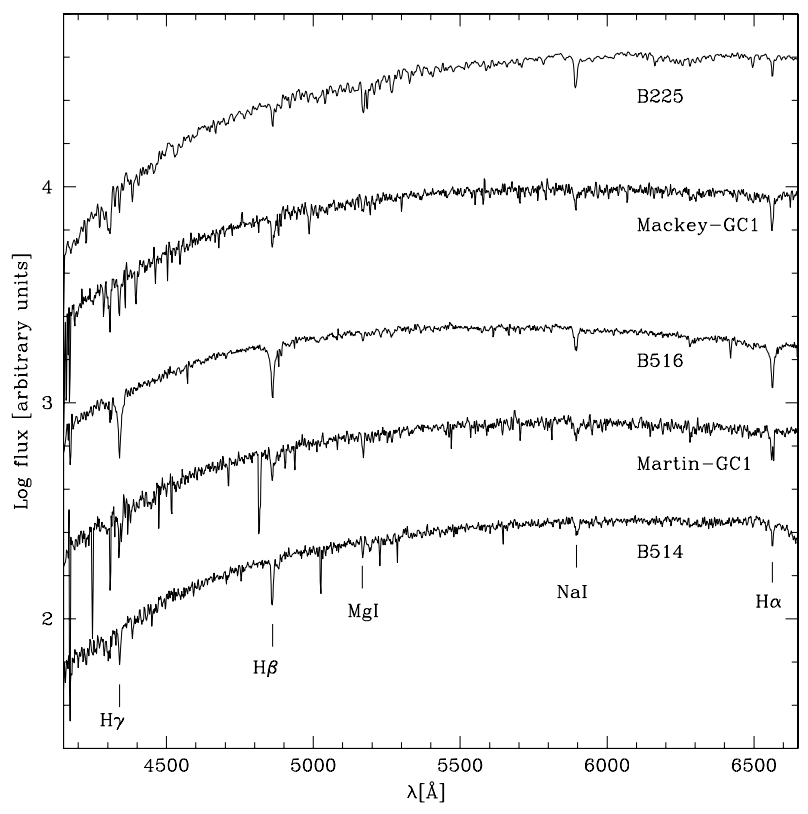

Fig. 5. BFOSC spectra of target clusters. From top to bottom, the RV template B225, a well known metal-rich cluster, reported here for comparison, Mackey-GC1, B516, Martin-GC1, and B514, a remote old and metal poor cluster (G05; G06b). The main spectral features have been labeled. Note the prominent Balmer's lines in the spectrum of B516.

is a spurious extended object due to the superposition of two or more unrelated stars. The $R$ parameter is not extreme $(R=1.09)$. In Fig. 1, B516 is the open pentagon with the highest value of $\log F$ : the point is outside the "star strip", but not so clearly on the "extended object" branch of the diagram as the other clusters studied here. Cohen et al. (2006) have demonstrated that some objects classified as M 31 BLCC because of their strong $\mathrm{H}_{\beta}$ and their RV typical of M 31 cluster are in fact asterisms, i.e. chance superpositions of stars residing in the star-forming thin disc of M 31. This kind of spurious clusters can be encountered only in the high-surface brightness inner regions of the M 31 disc, where the strong stellar crowding makes relatively likely the possibility of finding several bright M 31 stars within a very small angle $\left(\lesssim 5^{\prime}\right)$. This cannot be the case of B516, that lies at $\sim 4.8^{\circ}$ $\left(R_{\mathrm{p}} \simeq 65 \mathrm{kpc}\right)$ from the center of the galaxy, where no sign of the disc of M 31 is visible. It remains the possibility that B516 is a (or a blend of) Galactic F or A spectral type star(s). Such star should likely be a member of the Galactic Thin Disc since these early types are very rare in the Thick Disc and in the Halo.

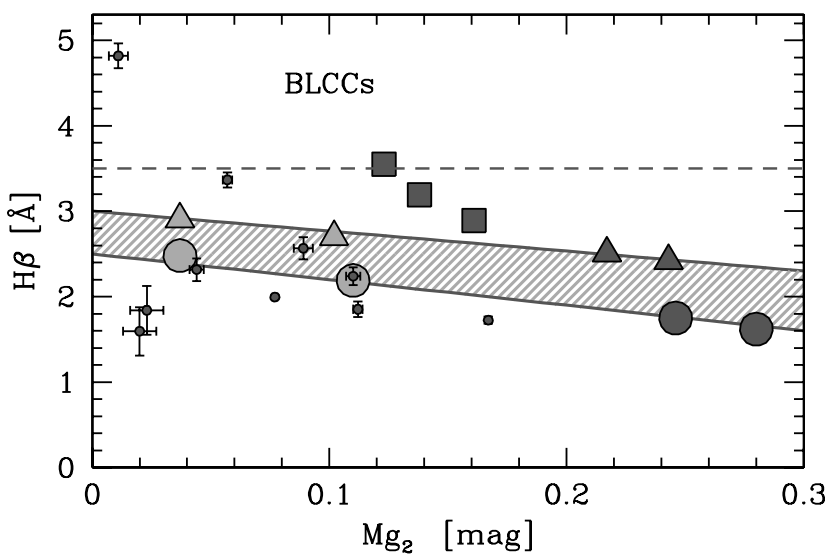

Fig. 6. The $\mathrm{H}_{\beta}$ and $\mathrm{Mg}_{2}$ indices of the observed clusters (Table 3, small filled circles with error bars) are compared with the predictions of the models by Buzzoni et al. (1992). The large circles correspond to the model of a Simple Stellar Population (SSP) of age 15 Gyr and metallicity $[\mathrm{Fe} / \mathrm{H}]=-2.27,-1.27,-0.25,0.0$, from left to right, with red Horizontal Branch morphology. The triangles correspond to the same model at the same metallicities but with an intermediate HB morphology, instead. Therefore, the shaded area encloses the region of the plane that is expected to be populated by old globular clusters. The filled squares correspond to a model of age $2 \mathrm{Gyr}$, with metallicity $[\mathrm{Fe} / \mathrm{H}]=-0.25,0.0,+0.3$, from left to right respectively. The realm of the Blue Luminous Compact Clusters (Fusi Pecci et al. 2005) is marked by a long dashed line and labeled. The cluster with the highest $\mathrm{H}_{\beta}$ is B516 and the cluster with the highest $\mathrm{Mg}_{2}$ is B519.

The synthetic sample of Galactic stars in the foreground of M 31 that we extracted from the Robin et al. (2003) model and we analyzed in G06a does not contain any Thin Disc star having $V_{\mathrm{r}}<-150 \mathrm{~km} \mathrm{~s}^{-1}$; actually, only $1 \%$ of the whole sample have $V_{\mathrm{r}}<-150 \mathrm{~km} \mathrm{~s}^{-1}$. In the same synthetic sample, Thick Disc stars have $\left\langle V_{\mathrm{r}}\right\rangle \simeq-53 \mathrm{~km} \mathrm{~s}^{-1}$ and dispersion $\sigma \simeq 54 \mathrm{~km} \mathrm{~s}^{-1}$; the rare Halo stars have $\left\langle V_{\mathrm{r}}\right\rangle \simeq-196 \mathrm{~km} \mathrm{~s}^{-1}$ and $\sigma \simeq 96 \mathrm{~km} \mathrm{~s}^{-1}$, hence the RV of B516 is not strictly incompatible with these Galactic components. The Monoceros Ring substructure (Yanny et al. 2003; Ibata et al. 2003) crosses the line of sight to M 31 and includes A, F stars; however Martin et al. (2006a) have found that Monoceros Ring stars in this direction have mean velocity $\left\langle V_{\mathrm{r}}\right\rangle \simeq-75 \mathrm{~km} \mathrm{~s}^{-1}$ and dispersion $\sigma \simeq 26 \mathrm{~km} \mathrm{~s}^{-1}$, which seem incompatible with the velocity of B516.

Given the scenario described above, a field Blue Straggler belonging to the Galactic Halo (see Carney et al. 2005, and 


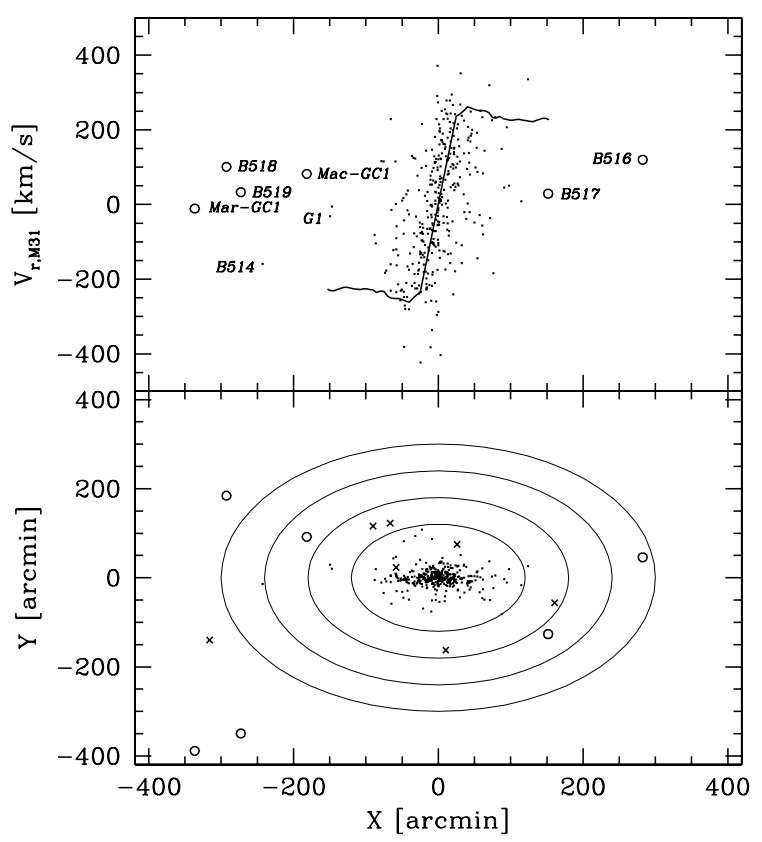

Fig. 7. Upper panel: velocity distribution along the major axis for already confirmed M 31 GCs (small points), and for remote clusters studied in the present paper (open circles). The line is the HI rotation curve from Carignan et al. (2006). $V_{\mathrm{r}, \mathrm{M} 31}$ is the line-of-sight velocity in the Andromeda-centric reference system. Lower panel: position of the clusters in the sky in the Andromeda-centric reference system; open circles represent remote clusters discovered in our survey, " $x$ " symbols mark clusters discovered by other teams. The overplotted concentric circles have radius $2,3,4$, and 5 degrees.

references therein) may be the more likely alternative explanation for the nature of B516.

In conclusion, the formal application of the criteria adopted in G06a would lead to classify B516 as a genuine M $31 \mathrm{GC}$; if this were the case we should also conclude that B516 is a young bright cluster as those described by Fusi Pecci et al. (2005). The occurrence of a young cluster so far away from the regions of M 31 where the star formation is presently ongoing is unexpected; since neither the RV, nor the available imaging can completely exclude the possibility that B516 is a foreground source we maintain a doubt on the final classification of this object, until further observations will clarify the issue.

\section{The population of remote GCs of M 31}

In Fig. 7 we show the velocity (upper panel) and spatial (lower panel) distribution of the confirmed M 31 GC from the RBC and of the recently discovered remote clusters. The HI rotation curve from Carignan et al. (2006) is also plotted in the upper panel, as a reference. The sample of remote clusters currently available for kinematical analysis does not show any clear correlation between position and $\mathrm{M}$ 31-centric velocity ( $\left.V_{\mathrm{r}, \mathrm{M} 31}\right)$. This seems consistent with the association of these clusters to the non-rotating very extended and metal-poor stellar halo that has been recently found in M 31 (Kalirai et al. 2006; Chapman et al. 2006). The velocity dispersion computed from the six objects listed in Table 2 plus B514 is $\sigma=94 \pm 46 \mathrm{~km} \mathrm{~s}^{-1}$, in agreement with what found by Chapman et al. (2006) for the stellar halo $\left(\sigma \simeq 126 \mathrm{~km} \mathrm{~s}^{-1}\right)$. A detailed analysis of the kinematics of the M 31 GC system as a whole is beyond the scope of the

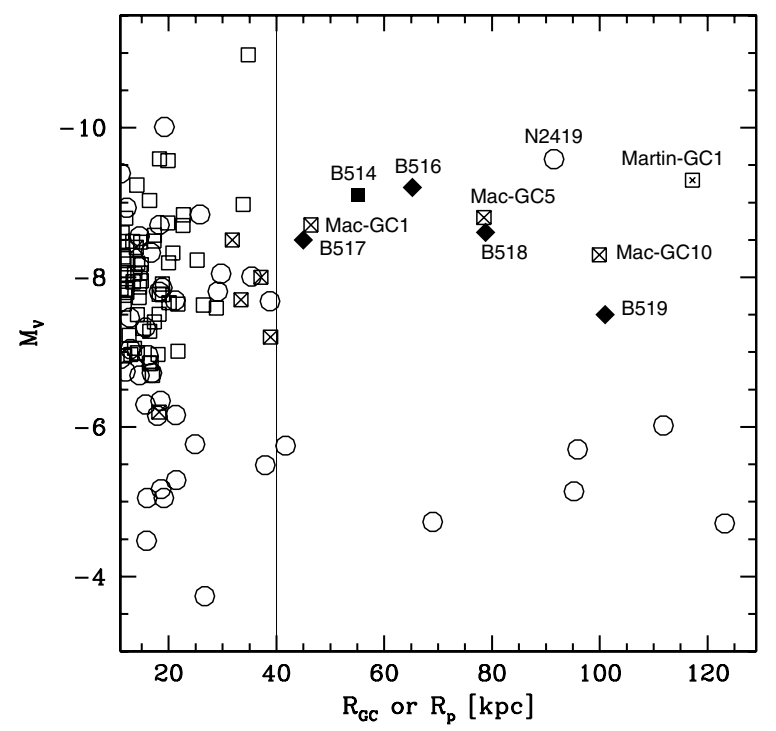

Fig. 8. Absolute integrated $V$ magnitude versus galactocentric distance (or projected galactocentric distance) for MW (open circles) and M 31 globular clusters (open squares). The crossed squares are the new clusters recently presented by Mackey et al. (2007); the open square with the small cross is the remote Martin et al.'s GC1; the filled square is B514 (G05; G06b; Federici et al. 2007); the filled diamonds are the newly identified remote clusters. The bright clusters at $R_{\mathrm{GC}}>40 \mathrm{kpc}$ have been labeled. The vertical line marks $R_{\mathrm{GC} / \mathrm{p}}=40 \mathrm{kpc}$.

present contribution and it is demanded to the end of our survey: it is quite clear from Fig. 7 that the (presumably) high incompleteness of the sample prevents any further speculation.

At the present stage, the most interesting characteristic of our (scanty) sample of remote M 31 GCs emerges from the comparison with the case of the Milky Way and is illustrated in Fig. 8 (see also Mackey et al. 2007). It is quite clear that the outskirts of M 31 host a significant population of bright clusters $\left(M_{V} \leq-7.5\right)$ whose only Galactic counterpart is NGC 2419, indeed a quite peculiar cluster (see Mackey \& van den Bergh 2005; Federici et al. 2007, and references therein). The joint luminosity distribution of remote clusters of both M 31 and the MW appears curiously bimodal, with a "faint" population $\left(M_{V} \sim-5\right)$ dominated by MW clusters and a "bright" population $\left(M_{V} \sim-8\right)$, constituted by M 31 clusters plus NGC 2419, with a wide gap in the middle $\left(M_{V} \sim-7\right)$. Note that while "faint" clusters are (probably) missing in the M 31 sample for obvious reasons of incompleteness, the lack of "bright" and intermediate luminosity clusters in the MW is clearly a real effect. The difference between the remote GC populations of the two galaxies is even more striking if one takes into account that the M 31 sample is clearly very far from complete (see Sect. 1.1.1): several bright remote clusters are probably still to be discovered.

At present, there is no obvious explanation for the luminosity distribution of remote clusters shown in Fig. 8. F07 pointed out that some of the newly discovered remote M 31 GCs have half-light radii that are too large for their luminosity with respect to ordinary globulars, resembling the nuclei of dwarf elliptical galaxies instead. Mackey \& van den Bergh (2005) noted that all the clusters having this characteristic (as, for instance NGC 2419) have been proposed in the past, for various reason, as the possible remnants of nucleated dwarf galaxies. If part of the bright remote clusters of Fig. 8 are the survived nuclei of disrupted galaxies, the difference between the MW and M 31 populations can be possibly interpreted in terms of initial conditions, that is, for example, it may have been determined by a 
difference in the luminosity function and/or in the distribution of morphological types of the original building blocks from which the two galaxies were assembled.

In any case, it is quite clear that the study of these remote clusters may reveal us fundamental pieces of information about the early stage of the formation of the Milky Way and M 31.

Acknowledgements. We thank the referee, P. Barmby, for her useful and prompt report. We are grateful to N.F. Martin and A.P. Huxor for their kind collaboration in cross-checking our results. A.B. acknowledges the support by INAF through the INAF/PRIN05 grants 1.06.08.02 and 1.06.08.03. We are grateful to the staff of the Telescopio Nazionale Galileo and Loiano Observatory for their kind assistance during the observing runs. This research has made use of the VizieR catalogue access tool, CDS, Strasbourg, France, and of NASA's Astrophysics Data System. The Digitized Sky Surveys were produced at the Space Telescope Science Institute under U.S. Government grant NAG W-2166.

\section{References}

Barmby, P., Huchra, J. P., \& Brodie, J. P. 2001, AJ, 121, 1482

Barmby, P., Holland, S., \& Huchra, J. P. 2002, AJ, 123, 1937

Barmby, P., McLaughlin, D. E., Harris, W. E., Harris., G. L. H., \& Forbes, D. A. 2007, AJ, 133, 2764

Bertin, E., \& Arnouts, S. 1996, A\&AS, 117, 393

Brodie, J. P., \& Strader, J. 2006, ARA\&A, 44, 193

Buzzoni, A., Gariboldi, G., \& Mantegazza, L. 1992, AJ, 103, 1814

Carignan, C., Chemin, L., Hutchmeier, W. K., \& Lockman, F. J. 2006, ApJ, 641, L109

Carney, B. W., Latham, D. W., \& Laird, J. B. 2005, AJ, 129, 466

Cohen, J. G., Matthews, K., \& Cameron, P. B. 2006, ApJ, 634, L45

Chapman, S. C., Ibata, R., Lewis, G. F., et al. 2006, ApJ, 653, 255

Dubath, P., \& Grillmair, C. J. 1997, A\&A, 321, 379

Federici, L., Bellazzini, M., Galleti, S., et al. 2007, A\&A, in press (F07)

Fusi Pecci, F., Bellazzini, M., Buzzoni, A., et al. 2005, AJ, 130, 554

Galleti, S., Federici, L., Bellazzini, M., Fusi Pecci, F., \& Macrina, S. 2004, A\&A, 416, 917 (G04)

Galleti, S., Bellazzini, M., Federici, L., \& Fusi Pecci, F. 2005, A\&A, 436, 535 (G05)
Galleti, S., Federici, L., Bellazzini, M., Buzzoni, A., \& Fusi Pecci, F. 2006a, A\&A, 456, 985 (G06a)

Galleti, S., Federici, L., Bellazzini, M., Buzzoni, A., \& Fusi Pecci, F. 2006b, ApJ, 650, L107 (G06b)

Gualandi, R., \& Merighi, R. 2001, Manual Reports, http://www.bo.astro.it/ loiano/TechPage/pagine/ BfoscManualTechPage/BfoscManual.htm

Harris, W. E. 1996, AJ, 112, 1487

Hubble, E. P. 1932, ApJ, 76, 44

Huxor, A., Tanvir, N. R., Irwin, M. J., in Satellites and Tidal tails, ed. F. Prada, D. Martinez-Delgado and T. Mahoney, (S. Francisco: ASP), ASP Conf. Ser. 2004, 327, 118 (H04)

Huxor, A. P., Tanvir, N. R., Irwin, M. J., et al. 2005, MNRAS, 360, 1007

Ibata, R., Irwin, M. J., Lewis, G., Ferguson, A. M. N., \& Tanvir, N. R. 2001, Nature, 412, 49

Ibata, R., Irwin, M. J., Lewis, G., \& Ferguson, A. M. N., \& Tanvir, N. R. 2003, MNRAS, 340, 21

Ibata, R., Chapman, S., \& Ferguson, A. M. N. 2004, MNRAS, 351, 117

Kalirai, J. S., Gilbert, K. M., \& Guhathakurta, P. 2006, ApJ, 648, 389

Mackey, A. D., \& van den Bergh, S. 2005, MNRAS, 360, 631

Mackey, A. D., Huxor, A., Ferguson, A. M. N., et al. 2007, ApJ, 655, L85

Martin, N. F., Irwin, M. J., Ibata, R. A., et al. 2006a, MNRAS, 367, L69

Martin, N. F., Ibata, R. A., Irwin, M. J., et al. 2006b, MNRAS, 371, 1983 (M06b)

McConnachie, A. W., Irwin, M. J., Ferguson, A. M. N., et al. 2005, MNRAS, 356,979

Mochejska, B. J., Kaluzny, J., Krockenberger, M., Sasselov, D. D., \& Stanek, K. Z. 1998, Acta Astron., 48, 455

Paturel, G., Petit, C., Prugniel, Ph., Theureau, G., et al. 2003, A\&A, 412, 245

Peterson, R. C. 1989, in Dynamics of dense stellar systems (Cambridge, England and New York: Cambridge University Press), 161

Puzia, T. H., Perrett, K. M., \& Bridges, T. J. 2005, A\&A, 434, 909

Renzini, A., \& Fusi Pecci, F. 1988, ARA\&A, 26, 199

Robin, A. C., Reylè, C., Derriére, S., \& Picaud, S. 2003, A\&A, 409, 523

Schlegel, D. J., Finkbeiner, D. P., \& Davis, M. 1998, ApJ, 500, 525

Skrutskie, M. F., Cutri, R. M., Stirning, R., et al. 2006, AJ, 131, 1163 (2MASS)

Thomas, D., Maraston, C., \& Bender, R. 2003, MNRAS, 339, 897

Trager, S. C., Worthey, G., Faber, S. M., Burstein, D., \& González, J. J. 1998, ApJS, 116, 1

Tonry, J., \& Davis, M. 1979, AJ, 84, 1511

Yanny, B., Newberg, H. J., Grebel, E. K., et al. 2003, ApJ, 588, 824

Zacharias, N., Monet, D. G., \& Levine, S. E. 2004, BAAS, 2054815 (NOMAD Catalog) 
S. Galleti et al.: A spectroscopic survey of globular clusters in M31. II., Online Material p 1

\section{Online Material}




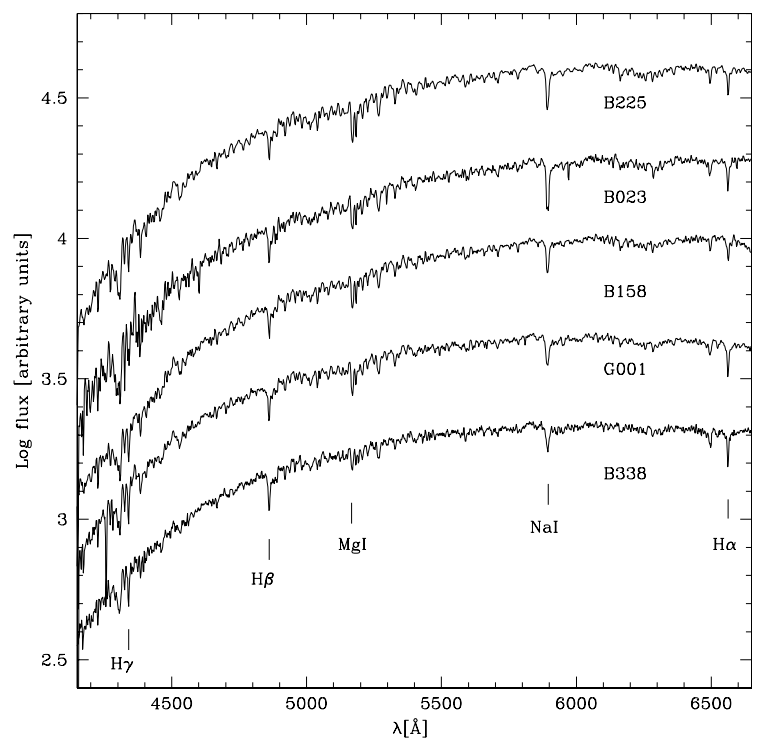

Fig. A.1. BFOSC spectra of template clusters. From top to bottom, the RV templates B225, B023, G001, B158, B338 cluster (G05, G06b). The main spectral features have been labeled. The typical $\mathrm{S} / \mathrm{N}$ is $\sim 100$ for $\lambda>4700 \AA$.

\section{Appendix A: Master RV templates for BFOSC}

In the past few years we had several observational runs dedicated to the acquisition of BFOSC spectra of M 31 clusters at the Cassini telescope. In all the cases we adopted the same set up described here and during every night of observation we acquired at least one spectrum of a bright well-known M 31 cluster for which a high-accuracy estimate of RV is available (from Peterson 1989 or Dubath \& Grillmair 1997, see G06a), to be used as template for the cross correlation. In this way we have assembled a conspicuous number of relatively high $\mathrm{S} / \mathrm{N}$ spectra of template clusters. We have verified that in all cases the spectra of the same template taken in different runs gave null velocity difference once cross-correlated, hence the velocity scale of BFOSC seems very stable. Given all the above, we decided to stack all the spectra of a given template cluster (taken in different nights/runs) to obtain master spectra with the highest possible $\mathrm{S} / \mathrm{N}$ ratio. The use of stacked spectra of a set of templates would significantly improve the accuracy of the RV estimate of target clusters.

Here we present the list of Master RV templates that has been used for the present study (Table A.1); the stacked spectra are shown in Fig. A.1.

In future BFOSC runs we will acquire spectra of templates during each observing night for the following purposes:

1. to verify that the acquired spectra are in the same velocity scale of the Master RV templates;

2. to obtain more spectra to stack into the Masters;

3. to include more clusters in the list of the Master RV templates.

The final goal of the project is to assemble high $\mathrm{S} / \mathrm{N}$ master spectra for $\sim 10 \mathrm{RV}$ templates of various metallicities, to maximize the return of future spectroscopic campaigns at the Cassini telescope.
Table A.1. Master RV template clusters (BFOSC).

\begin{tabular}{ccrrcc}
\hline \hline Name & $\begin{array}{c}V \\
\mathrm{mag}\end{array}$ & $\begin{array}{r}V_{\mathrm{r}} \pm \epsilon_{V \mathrm{r}} \\
\mathrm{km} \mathrm{s}^{-1}\end{array}$ & $\begin{array}{r}N(\mathrm{oss}) \\
\mathrm{dex}\end{array}$ & {$[\mathrm{Fe} / \mathrm{H}]$} & $S / N$ \\
\hline B023 & 14.22 & $-451 \pm 5$ & 2 & - & 140 \\
B158 & 14.70 & $-187 \pm 1$ & 5 & -0.70 & 200 \\
B225 & 14.15 & $-165 \pm 1$ & 13 & -0.45 & 500 \\
B338 & 14.25 & $-274 \pm 3$ & 2 & -1.17 & 90 \\
G001 & 13.21 & $-332 \pm 3$ & 3 & -1.02 & 115 \\
\hline
\end{tabular}

Metallicities are taken from Puzia et al. 2005. We have transformed $[\mathrm{Z} / \mathrm{H}]$ into $[\mathrm{Fe} / \mathrm{H}]$ from the equation: $[\mathrm{Fe} / \mathrm{H}]=[\mathrm{Z} / \mathrm{H}]-0.94[\alpha / \mathrm{Fe}]$ by Thomas et al. 2003

\section{Appendix B: Updates to the RBC}

As a duty cycle operation to maintain the RBC as updated as possible, we search the literature for new classification and data on M 31 GCs and we periodically search the HST archive for intentional or serendipitous images of M 31 CGCs that can potentially reveal the true nature of some candidate.

Here we report on the results of the careful visual inspection of archival HST images of 164 objects already included in the RBC, irrespectively of their current classification, as it may happen that a very high resolution image supersedes even a spectroscopic classification (see G06a). The results are summarized in Table B.3 where we report: the name of the target (Col. 1); the spectroscopic classification, as defined in G04: C - cluster, G - galaxy, S -star, H - H II region (Col. 2); previous classification via high resolution imaging, as defined in G04: C - cluster, G galaxy, S - star, H - H II region, A - asterism (Col. 3); the classification obtained from the visual inspection of HST images in the present work ${ }^{10}$ (Col. 4$)$; the final classification flag adopted in the new version of the RBC (1: confirmed GC, 2: GC candidate, 3: controversial object, 4: confirmed galaxy, 5: confirmed $\mathrm{H}$ II region, 6: confirmed star, 7: asterism) (Col. 5); the Proposal Id number (Col. 6); the instrument (Col. 7); the passband of the considered image (Col. 8); the image name (Col. 9); the exposure time (Col. 10).

Most of the inspected objects (130) were already classified in the RBC (as star, cluster, galaxy etc), but only 43 objects were previously classified with high resolution imaging. 34 objects were just CGCs that are classified here for the first time. Two of these candidates turn out to be genuine globular clusters (B202 and AU010), B004D and B328D are clearly background galaxies, while all the other candidates are stars or blended groups of stars or asterisms.

The controversial candidates B140 and B366 are clearly resolved into stars in the inspected images, hence they are genuine clusters, while B175 is clearly a star. B253 and B034D, classified as genuine globular clusters by G06a based on their radial velocity are recognized as stars in the considered images. B147 was classified as a star by Dubath \& Grillmair (1997), based on its velocity dispersion: the HST images unambiguously reveal that this is a well resolved star cluster, as recently pointed out also by Barmby et al. (2007).

While inspecting this huge batch of HST images, we identified four new candidates, listed in Table B.1 and christened B521, B522, B523, and B524. The images clearly reveal these

\footnotetext{
10 Classifications followed by a question mark indicates that a completely firm conclusion on the nature of an object cannot be reached based on the considered image alone. For example, the classification "globular cluster" indicates an object clearly resolved into stars, "globular cluster?" indicates the case of a roundish extended object resembling a cluster but NOT clearly resolved into stars.
} 
Table B.1. Newly discovered globular clusters candidates.

\begin{tabular}{lrrrrrrr}
\hline \hline Name & c & RA & Dec & Camera & Filter & Dataset & Exptime \\
\hline B521 & 2 & $00: 41: 41.67$ & $+40: 52: 01.41$ & ASC/WFC & $F 606 W$ & j96q06010 & 3250 \\
B522 & 2 & $00: 41: 50.94$ & $+40: 52: 48.29$ & ASC/WFC & $F 606 W$ & j96q06010 & 3250 \\
B523 & 2 & $00: 42: 46.28$ & $+41: 18: 32.41$ & ACS/WFC & $F 435 W$ & j8vp05010 & 2200 \\
B524 & 2 & $00: 42: 55.68$ & $+41: 03: 11.22$ & ACS/WFC & $F 775 W$ & j8hoiiq8q & 624 \\
\hline
\end{tabular}

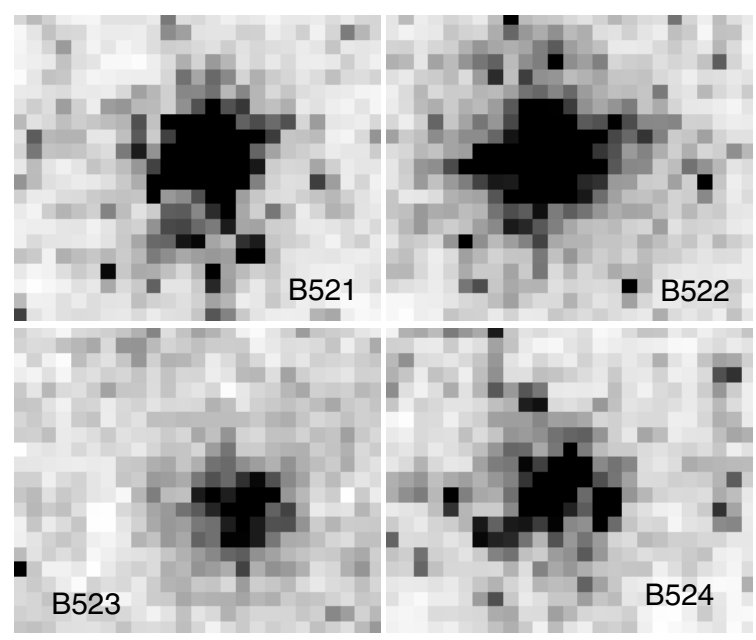

Fig. B.1. New globular cluster candidates found in HST images: B521, B522, B523 and B524. The size of the post-stamp images shown here is $\sim 5^{\prime \prime} \times 5^{\prime \prime}$.

Table B.2. Radial velocities of globular cluster candidates drawn from the RBC.

\begin{tabular}{lcrrr}
\hline \hline Name & $V_{\mathrm{r}}$ & $\pm \epsilon_{V r}$ & Source & Classification \\
\hline B052 & 30060 & 65 & R3 LOI & galaxy \\
B062 & 38180 & 53 & R3 LOI & galaxy \\
B503 & 29330 & 60 & $27 / 09 / 05$ & galaxy \\
B285D & 14415 & 32 & R2 LOI & galaxy \\
B329D & 32141 & 51 & R2 LOI & galaxy \\
G289 & -53 & 7 & R2 LOI & star? \\
G295 & -74 & 12 & R2 LOI & star? \\
\hline
\end{tabular}

objects as non-stellar: however they are faint and not clearly resolved into stars, hence a spectrum is required to ultimately assess their classification (see Fig. B.1).

During the observing runs at the Cassini Telescope (Sect. 2.2), in addition to the remote CGCs listed in Table 1, we have obtained radial velocities also for 7 candidates already listed in the RBC. The results and classifications for these CGCs are listed in Table B.2. All of these candidates turned out to be galaxies or foreground stars.

All the present observational material has been consistently implemented to update the RBC, available on line in its latest V3.0 release. Future minor updates of the cata$\log$ will be described and commented in the RBC web page (http://www.bo.astro.it/M 31/) where the catalog can be retrieved in form of ASCII files. 
S. Galleti et al.: A spectroscopic survey of globular clusters in M31. II., Online Material p 4

Table B.3. Globular clusters candidates from HST images.

\begin{tabular}{|c|c|c|c|c|c|c|c|c|c|}
\hline Name & $\overline{\mathrm{S}}$ & $\overline{\mathrm{V}}$ & Classification & $\overline{\mathrm{c}}$ & Prop. ID & Camera & Filter & $\overline{\text { Dataset }}$ & Exptime \\
\hline B008 & $\mathrm{C}$ & & globular cluster & 1 & 10407 & ASC/WFC & $F 606 W$ & j96q07010 & 3250 \\
\hline B010 & $\mathrm{C}$ & & globular cluster & 1 & 10407 & ASC/WFC & $F 606 W$ & j96q07010 & 3250 \\
\hline B013 & $\mathrm{C}$ & & globular cluster & 1 & 9767 & ACS/WFC & $F 814 W$ & j80420c9q & 774 \\
\hline B023 & $\mathrm{C}$ & $\mathrm{C}$ & globular cluster & 1 & 9719 & ACS/HRC & F606W & j8pz02010 & 2020 \\
\hline B037 & $\mathrm{C}$ & $\mathrm{C}$ & globular cluster & 1 & 10260 & ACS/WFC1 & F606W & j8z003010 & 2370 \\
\hline B041 & $\mathrm{C}$ & & globular cluster & 1 & 10260 & ACS/WFC1 & F606W & j8z003010 & 2370 \\
\hline B042 & $\mathrm{C}$ & & globular cluster & 1 & 10260 & ACS/WFC1 & F606W & j8z006010 & 2370 \\
\hline B049 & $\mathrm{C}$ & & globular cluster & 1 & 10407 & ASC/WFC & F606W & j96q06010 & 3250 \\
\hline B056 & $\mathrm{C}$ & & globular cluster? & 1 & 9087 & ACS/WFC1 & $F 435 W$ & j6d509020 & 2100 \\
\hline B057 & $\mathrm{C}$ & & globular cluster & 1 & 10407 & ASC/WFC & F606W & j96q06010 & 3250 \\
\hline B061 & $\mathrm{C}$ & $\mathrm{C}$ & globular cluster & 1 & 10260 & ACS/WFC1 & F606W & j8z008010 & 2370 \\
\hline B063 & $\mathrm{C}$ & $\mathrm{C}$ & globular cluster & 1 & 10260 & ACS/WFC1 & F606W & j8z008010 & 2370 \\
\hline B068 & $\mathrm{C}$ & & globular cluster? & 1 & 9087 & ACS/WFC1 & $F 435 W$ & j6d509020 & 2100 \\
\hline B082 & $\mathrm{C}$ & & globular cluster & 1 & 10260 & ACS/WFC1 & $F 606 W$ & j8z004010 & 2370 \\
\hline B086 & $\mathrm{C}$ & & globular cluster & 1 & 10094 & ACS/WFC & $F 814 W$ & j92t46qpq & 824 \\
\hline B088 & $\mathrm{C}$ & & globular cluster & 1 & 10260 & ACS/WFC1 & F606W & j8z007010 & 2370 \\
\hline B090 & $\mathrm{C}$ & & globular cluster? & 1 & 10260 & ACS/WFC1 & F606W & j8z004010 & 2370 \\
\hline B091 & $\mathrm{C}$ & $\mathrm{C}$ & globular cluster? & 1 & 10273 & ACS/WFC & $F 814 W$ & j92gb3dnq & 502 \\
\hline B093 & $\mathrm{C}$ & $\mathrm{C}$ & globular cluster & 1 & 10273 & ACS/WFC & $F 814 W$ & j92gb3dnq & 502 \\
\hline B094 & $\mathrm{C}$ & & globular cluster & 1 & 10273 & ACS/WFC & $F 814 W$ & j92gb8vwq & 502 \\
\hline B102 & $\mathrm{C}$ & A & asterism & 7 & 10260 & ACS/WFC1 & F606W & j8z007010 & 2370 \\
\hline B103 & $\mathrm{C}$ & $\mathrm{C}$ & globular cluster & 1 & 10006 & ACS/WFC & $F 435 W$ & j8vp04010 & 2200 \\
\hline B104 & $\mathrm{C}$ & & globular cluster & 1 & 10006 & ACS/WFC & $F 435 W$ & j8vp04010 & 2200 \\
\hline B112 & $\mathrm{C}$ & $\mathrm{C}$ & globular cluster & 1 & 10006 & ACS/WFC & $F 435 W$ & j8vp04010 & 2200 \\
\hline B117 & $\mathrm{C}$ & & globular cluster? & 1 & 9087 & WFPC2/PC1 & $F 336 W$ & j6d50105r & 500 \\
\hline B119 & $\mathrm{C}$ & & globular cluster & 1 & 10006 & ACS/WFC & $F 435 W$ & j8vp04010 & 2200 \\
\hline B120 & & & star & 6 & 10006 & ACS/WFC & $F 435 W$ & j8vp04010 & 2200 \\
\hline B124 & $\mathrm{C}$ & $\mathrm{C}$ & globular cluster & 1 & 10006 & ACS/WFC & $F 435 W$ & j8vp03010 & 2200 \\
\hline B126 & $\mathrm{C}$ & $\mathrm{C}$ & globular cluster & 1 & 10006 & ACS/WFC & $F 435 W$ & j8vp02010 & 2200 \\
\hline B127 & $\mathrm{C}$ & $\mathrm{C}$ & globular cluster & 1 & 10006 & ACS/WFC & $F 435 W$ & j8vp03010 & 2200 \\
\hline B128 & & & globular cluster? & 2 & 10006 & ACS/WFC & $F 435 W$ & j8vp02010 & 2200 \\
\hline B130 & $\mathrm{C}$ & & globular cluster & 1 & 10273 & ACS/WFC & $F 814 W$ & j92gb4e7q & 502 \\
\hline B131 & $\mathrm{C}$ & $\mathrm{C}$ & globular cluster & 1 & 10006 & ACS/WFC & $F 435 W$ & j8vp03010 & 2200 \\
\hline B132 & & & globular cluster? & 2 & 10006 & ACS/WFC & $F 435 W$ & j8vp03010 & 2200 \\
\hline B134 & $\mathrm{C}$ & & globular cluster & 1 & 10118 & ACS/WFC & F660N & j8zs04040 & 2028 \\
\hline B140 & $\mathrm{C}$ & $\mathrm{G}$ & globular cluster & 1 & 10273 & ACS/WFC & $F 814 W$ & j92gb1fnq & 502 \\
\hline B144 & $\mathrm{C}$ & & globular cluster & 1 & 10118 & ACS/WFC & $F 814 W$ & j8zs05a6q & 507 \\
\hline B145 & & & globular cluster? & 2 & 10006 & ACS/WFC & $F 435 W$ & j8vp02010 & 2200 \\
\hline B146 & $\mathrm{C}$ & & globular cluster & 1 & 10118 & ACS/WFC & $F 814 W$ & $\mathrm{j} 8 \mathrm{zs} 05 \mathrm{a} 6 \mathrm{q}$ & 507 \\
\hline B147 & $\mathrm{S}$ & & globular cluster & 1 & 10260 & ACS/WFC1 & F606W & j8z005010 & 2370 \\
\hline B148 & $\mathrm{C}$ & $\mathrm{C}$ & globular cluster & 1 & 9087 & ACS/WFC1 & $F 435 W$ & j6d508010 & 2100 \\
\hline B151 & $\mathrm{C}$ & & globular cluster & 1 & 10260 & ACS/WFC1 & $F 606 W$ & j8z005010 & 2370 \\
\hline B152 & $\mathrm{C}$ & $\mathrm{C}$ & globular cluster & 1 & 9087 & ACS/WFC1 & $F 435 W$ & j6d508020 & 2100 \\
\hline B153 & $\mathrm{C}$ & & globular cluster & 1 & 9087 & ACS/WFC1 & $F 435 W$ & j6d508010 & 2100 \\
\hline B154 & $\mathrm{C}$ & $\mathrm{C}$ & globular cluster & 1 & 9087 & ACS/WFC1 & $F 435 W$ & j6d508010 & 2100 \\
\hline B155 & $\mathrm{C}$ & & globular cluster & 1 & 9480 & ACS/WFC & $F 775 W$ & j8hohleeq & 700 \\
\hline B156 & $\mathrm{C}$ & & globular cluster & 1 & 9480 & ACS/WFC & $F 775 W$ & j8hohleeq & 700 \\
\hline B158 & $\mathrm{C}$ & & globular cluster & 1 & 9719 & ACS/HRC & F606W & j8pz02010 & 2020 \\
\hline B159 & $\mathrm{C}$ & & globular cluster & 1 & 10006 & ACS/WFC & $F 435 W$ & j8vp08010 & 2200 \\
\hline B160 & $\mathrm{C}$ & & globular cluster & 1 & 9480 & ACS/WFC & $F 775 W$ & j8hohleeq & 700 \\
\hline B162 & $\mathrm{C}$ & $\mathrm{C}$ & globular cluster & 1 & 10006 & ACS/WFC & $F 435 W$ & j8vp08010 & 2200 \\
\hline B169 & $\mathrm{C}$ & & globular cluster & 1 & 10407 & ASC/WFC & F606W & j96q03010 & 3396 \\
\hline B171 & $\mathrm{C}$ & & globular cluster & 1 & 10407 & ASC/WFC & F606W & j96q03010 & 3396 \\
\hline B174 & $\mathrm{C}$ & & globular cluster & 1 & 10273 & ACS/WFC & $F 814 W$ & j92gb8vwq & 502 \\
\hline B175 & $\mathrm{C}$ & & star & 6 & 10407 & ASC/WFC & $F 606 W$ & j96q03010 & 3396 \\
\hline B185 & $\mathrm{C}$ & & globular cluster & 1 & 10407 & ASC/WFC & F606W & j96q03010 & 3396 \\
\hline B198 & $\mathrm{C}$ & & globular cluster & 1 & 10407 & ASC/WFC & $F 606 W$ & j96q05010 & 1840 \\
\hline B199 & $\mathrm{C}$ & & globular cluster & 1 & 9392 & WFPC2/PC1 & $F 606 W$ & u8f10201m & 2400 \\
\hline B201 & $\mathrm{C}$ & & globular cluster & 1 & 10273 & ACS/WFC & $F 814 W$ & j92gb6d3q & 502 \\
\hline B202 & & & globular cluster & 1 & 9392 & WFPC2/PC1 & F606W & $\mathrm{u} 8 \mathrm{f} 10201 \mathrm{~m}$ & 2400 \\
\hline
\end{tabular}


S. Galleti et al.: A spectroscopic survey of globular clusters in M31. II., Online Material p 5

Table B.3. continued.

\begin{tabular}{|c|c|c|c|c|c|c|c|c|c|}
\hline Name & $\overline{\mathrm{S}}$ & $\overline{\mathrm{V}}$ & Classification & $\overline{\mathrm{c}}$ & Prop. ID & Camera & Filter & Dataset & Exptime \\
\hline B203 & $\mathrm{C}$ & & globular cluster & 1 & 10407 & ASC/WFC & $F 606 W$ & j96q05010 & 1840 \\
\hline B206 & $\mathrm{C}$ & & globular cluster & 1 & 10407 & ASC/WFC & $F 606 W$ & j96q05010 & 1840 \\
\hline B213 & $\mathrm{C}$ & & globular cluster & 1 & 10407 & ASC/WFC & $F 606 W$ & j96q05010 & 1840 \\
\hline B215 & $\mathrm{C}$ & & globular cluster & 1 & 10407 & ASC/WFC & $F 606 W$ & j96q05010 & 1840 \\
\hline B220 & $\mathrm{C}$ & & globular cluster & 1 & 10407 & ASC/WFC & F606W & j96q02010 & 1860 \\
\hline B224 & $\mathrm{C}$ & & globular cluster & 1 & 10407 & ASC/WFC & $F 606 W$ & j96q02010 & 1860 \\
\hline B225 & $\mathrm{C}$ & $\mathrm{C}$ & globular cluster & 1 & 9719 & ACS/HRC & F606W & j8pz02010 & 2020 \\
\hline B231 & $\mathrm{C}$ & & globular cluster & 1 & 10407 & ASC/WFC & F606W & j96q04010 & 3315 \\
\hline B234 & $\mathrm{C}$ & & globular cluster & 1 & 10407 & ASC/WFC & F606W & j96q04010 & 3315 \\
\hline B253 & $\mathrm{C}$ & & stars & 6 & 10407 & ASC/WFC & F606W & j96q06010 & 3250 \\
\hline B257 & & & globular cluster? & 2 & 9087 & ACS/WFC1 & $F 435 W$ & j6d509020 & 2100 \\
\hline B261 & & $\mathrm{S}$ & stars & 6 & 10006 & ACS/WFC & $F 435 W$ & j8vp04010 & 2200 \\
\hline B264 & $\mathrm{S}$ & $\mathrm{C}$ & globular cluster? & 2 & 10006 & ACS/WFC & $F 435 W$ & j8vp03010 & 2200 \\
\hline B353 & $\mathrm{S}$ & & blank & 6 & 10006 & ACS/WFC & $F 435 W$ & j8vp03010 & 2200 \\
\hline B366 & $\mathrm{C}$ & & globular cluster & 1 & 10407 & ASC/WFC & $F 606 W$ & j96q01010 & 1850 \\
\hline B367 & $\mathrm{C}$ & & globular cluster & 1 & 10407 & ASC/WFC & F606W & j96q01010 & 1850 \\
\hline B407 & $\mathrm{C}$ & $\mathrm{C}$ & globular cluster & 1 & 9458 & WFPC2 & $F 814 W$ & $\mathrm{u} 8 \mathrm{db} 0701 \mathrm{~m}$ & 1100 \\
\hline B458 & $\mathrm{C}$ & & $\mathrm{GC}=\mathrm{B} 031 \mathrm{D}$ & 1 & 10407 & ASC/WFC & F606W & j96q06010 & 3250 \\
\hline B465 & & & blank & 2 & 10118 & ACS/WFC & $F 814 W$ & j8zs05a6q & 507 \\
\hline B515 & & $\mathrm{C}$ & globular cluster & 1 & 10260 & ACS/WFC1 & $F 606 W$ & j8z007010 & 2370 \\
\hline B004D & & & galaxy & 4 & 10407 & ASC/WFC & F606W & j96q07010 & 3250 \\
\hline B034D & $\mathrm{C}$ & & star & 6 & 10407 & ASC/WFC & F606W & j96q06010 & 3250 \\
\hline $\mathrm{B} 056 \mathrm{D}$ & & $\mathrm{C}$ & globular cluster & 1 & 10260 & ACS/WFC1 & F606W & j8z007010 & 2370 \\
\hline B058D & & & stars & 6 & 10273 & ACS/WFC & $F 814 W$ & j92gb3dnq & 502 \\
\hline B059D & & & star & 6 & 10273 & ACS/WFC & $F 814 W$ & j92gb3cvq & 502 \\
\hline B062D & & & galaxy? & 2 & 10273 & ACS/WFC & $F 814 W$ & j92gb3cvq & 502 \\
\hline B065D & & & star & 6 & 10006 & ACS/WFC & $F 435 W$ & j8vp05010 & 2200 \\
\hline B074D & & $\mathrm{S}$ & blank & 6 & 10006 & ACS/WFC & $F 435 W$ & j8vp03010 & 2200 \\
\hline B075D & & $\mathrm{S}$ & star & 6 & 10006 & ACS/WFC & $F 435 W$ & j8vp03010 & 2200 \\
\hline B077D & & & stars & 6 & 9392 & ACS/WFC & F606W & $\mathrm{j} 8 \mathrm{f} 101 \mathrm{mtq}$ & 1000 \\
\hline B080D & & $\mathrm{S}$ & star & 6 & 10118 & ACS/WFC & $F 814 W$ & j8zs05a6q & 507 \\
\hline B081D & & & stars & 6 & 9480 & ACS/WFC & $F 775 W$ & j8hoiiq $8 \mathrm{q}$ & 624 \\
\hline B083D & & & star & 6 & 9480 & ACS/WFC & $F 775 W$ & j8hoiiq8q & 624 \\
\hline B085D & & & stars & 6 & 9392 & ACS/WFC & F606W & j8f101mtq & 1000 \\
\hline B086D & & $\mathrm{S}$ & star & 6 & 9392 & ACS/WFC & F606W & j8f101mtq & 1000 \\
\hline B087D & & & globular cluster? & 2 & 10273 & ACS/WFC & $F 814 W$ & j92gb1fnq & 502 \\
\hline B088D & & & globular cluster? & 2 & 9719 & ACS/WFC & $F 625 W$ & u8pz01020 & 1448 \\
\hline B090D & $\mathrm{C}$ & & globular cluster & 1 & 10118 & ACS/WFC & $F 814 W$ & j8zs05a6q & 507 \\
\hline B092D & & & stars? & 2 & 10006 & ACS/WFC & $F 435 W$ & j8vp02010 & 2200 \\
\hline B093D & & $\mathrm{S}$ & star & 6 & 9392 & ACS/WFC & F606W & $\mathrm{j} 8 \mathrm{f} 101 \mathrm{mtq}$ & 1000 \\
\hline B094D & & & stars & 6 & 10006 & ACS/WFC & $F 435 W$ & j8vp02010 & 2200 \\
\hline B112D & & & globular cluster? & 2 & 9719 & ACS/WFC & $F 625 W$ & u8pz03020 & 1448 \\
\hline $\mathrm{B} 255 \mathrm{D}$ & $\mathrm{C}$ & & globular cluster & 1 & 10407 & ASC/WFC & $F 606 W$ & j96q01010 & 1850 \\
\hline B316D & & & star & 6 & 9458 & WFPC2 & $F 814 W$ & $\mathrm{u} 8 \mathrm{db} 0801 \mathrm{~m}$ & 1100 \\
\hline B318D & & & star & 6 & 9458 & WFPC2 & $F 814 W$ & $\mathrm{u} 8 \mathrm{db} 0801 \mathrm{~m}$ & 1100 \\
\hline B328D & & & galaxy & 4 & 9458 & WFPC2 & $F 814 W$ & $\mathrm{u} 8 \mathrm{db} 0901 \mathrm{~m}$ & 1100 \\
\hline G137 & & $\mathrm{H}$ & HII region & 5 & 10260 & ACS/WFC1 & $F 606 W$ & j8z008010 & 2370 \\
\hline G204 & & & star & 6 & 9087 & ACS/WFC1 & $F 435 W$ & j6d508020 & 2100 \\
\hline V254 & & & HII region? & 2 & 10273 & ACS/WFC & $F 814 W$ & j92gb6d3q & 502 \\
\hline NB17 & $\mathrm{C}$ & & globular cluster? & 1 & 10006 & ACS/WFC & $F 435 W$ & j8vp05010 & 2200 \\
\hline NB18 & & & blank & 2 & 10006 & ACS/WFC & $F 435 W$ & j8vp05010 & 2200 \\
\hline NB21 & $\mathrm{C}$ & & globular cluster? & 1 & 10006 & ACS/WFC & $F 435 W$ & j8vp05010 & 2200 \\
\hline NB23 & & & globular cluster? & 2 & 10006 & ACS/WFC & $F 435 W$ & j8vp04010 & 2200 \\
\hline NB26 & $\mathrm{S}$ & & stars & 6 & 10006 & ACS/WFC & $F 435 W$ & j8vp02010 & 2200 \\
\hline NB28 & & & star & 6 & 10006 & ACS/WFC & $F 435 W$ & j8vp05010 & 2200 \\
\hline NB30 & & & star & 6 & 10006 & ACS/WFC & $F 435 W$ & j8vp03010 & 2200 \\
\hline NB32 & $\mathrm{S}$ & & stars & 6 & 10006 & ACS/WFC & $F 435 W$ & j8vp04010 & 2200 \\
\hline NB34 & & & stars? & 2 & 10118 & ACS/WFC & $F 814 W$ & j8zs05a6q & 507 \\
\hline NB35 & & & globular cluster? & 2 & 10006 & ACS/WFC & $F 435 W$ & j8vp04010 & 2200 \\
\hline NB36 & & & star & 6 & 10006 & ACS/WFC & $F 435 W$ & j8vp04010 & 2200 \\
\hline NB37 & & $\mathrm{S}$ & stars & 6 & 10006 & ACS/WFC & $F 435 W$ & j8vp04010 & 2200 \\
\hline
\end{tabular}


S. Galleti et al.: A spectroscopic survey of globular clusters in M31. II., Online Material p 6

Table B.3. continued.

\begin{tabular}{|c|c|c|c|c|c|c|c|c|c|}
\hline$\overline{\text { Name }}$ & $\overline{\mathrm{S}}$ & $\overline{\overline{\mathrm{V}}}$ & $\overline{\text { Classification }}$ & $\mathrm{c}$ & Prop. ID & Camera & Filter & $\overline{\overline{\text { Dataset }}}$ & $\overline{\text { Exptime }}$ \\
\hline NB39 & & & star? & 2 & 10006 & ACS/WFC & $F 435 W$ & j8vp03010 & 2200 \\
\hline NB40 & & & star & 6 & 10118 & ACS/WFC & F660N & j8zs04040 & 2028 \\
\hline NB42 & & & star & 6 & 10006 & ACS/WFC & $F 435 W$ & j8vp03010 & 2200 \\
\hline NB44 & & $\mathrm{S}$ & stars & 6 & 10006 & ACS/WFC & $F 435 W$ & j8vp05010 & 2200 \\
\hline NB45 & & $\mathrm{S}$ & stars & 6 & 10118 & ACS/WFC & F660N & j8zs04040 & 2028 \\
\hline NB49 & & $\mathrm{S}$ & stars & 6 & 10006 & ACS/WFC & $F 435 W$ & j8vp02010 & 2200 \\
\hline NB50 & & & star & 6 & 10006 & ACS/WFC & $F 435 W$ & j8vp02010 & 2200 \\
\hline NB52 & & & star & 6 & 10006 & ACS/WFC & F435W & j8vp02010 & 2200 \\
\hline NB55 & & & star & 6 & 10006 & ACS/WFC & $F 435 W$ & j8vp03010 & 2200 \\
\hline NB58 & & & star & 6 & 10006 & ACS/WFC & F435W & j8vp03010 & 2200 \\
\hline NB60 & & & blank & 2 & 10006 & ACS/WFC & $F 435 W$ & j8vp04010 & 2200 \\
\hline NB68 & $\mathrm{S}$ & & star & 6 & 10006 & ACS/WFC & $F 435 W$ & j8vp04010 & 2200 \\
\hline NB70 & $\mathrm{S}$ & & star & 6 & 10006 & ACS/WFC & $F 435 W$ & j8vp04010 & 2200 \\
\hline NB73 & & & stars & 6 & 10006 & ACS/WFC & $F 435 W$ & j8vp04010 & 2200 \\
\hline NB74 & $\mathrm{S}$ & & star & 6 & 10006 & ACS/WFC & $F 435 W$ & j8vp03010 & 2200 \\
\hline NB75 & & $S$ & star & 6 & 10006 & ACS/WFC & $F 435 W$ & j8vp03010 & 2200 \\
\hline NB79 & & & star & 6 & 10094 & ACS/WFC & $F 814 W$ & j92t46qpq & 824 \\
\hline NB89 & $\mathrm{C}$ & & globular cluster? & 1 & 10006 & ACS/WFC & F435W & j8vp03010 & 2200 \\
\hline NB90 & & & star & 6 & 10118 & ASC/WFC & F660N & j8zs04040 & 2028 \\
\hline NB92 & & $S$ & star? & 6 & 10006 & ACS/WFC & $F 435 W$ & j8vp02010 & 2200 \\
\hline NB94 & $\mathrm{S}$ & $S$ & star & 6 & 10006 & ACS/WFC & $F 435 W$ & j8vp03010 & 2200 \\
\hline NB95 & $\mathrm{S}$ & $\mathrm{S}$ & star & 6 & 10006 & ACS/WFC & $F 435 W$ & j8vp03010 & 2200 \\
\hline NB97 & & $S$ & star & 6 & 10118 & ACS/WFC & F660N & j8zs04040 & 2028 \\
\hline NB98 & & & star & 6 & 10006 & ACS/WFC & $F 435 W$ & j8vp02010 & 2200 \\
\hline NB99 & & $S$ & star & 6 & 10006 & ACS/WFC & $F 435 W$ & j8vp02010 & 2200 \\
\hline NB100 & & $\mathrm{S}$ & star & 6 & 10006 & ACS/WFC & $F 435 W$ & j8vp02010 & 2200 \\
\hline NB101 & & & star & 6 & 10006 & ACS/WFC & $F 435 W$ & j8vp02010 & 2200 \\
\hline NB102 & & & star & 6 & 10006 & ACS/WFC & $F 435 W$ & j8vp04010 & 2200 \\
\hline NB103 & & $S$ & star & 6 & 10118 & ACS/WFC & $F 814 W$ & $\mathrm{j} 8 \mathrm{zs} 05 \mathrm{a} 6 \mathrm{q}$ & 507 \\
\hline NB104 & & $S$ & star & 6 & 10006 & ACS/WFC & $F 435 W$ & j8vp02010 & 2200 \\
\hline NB105 & & & star & 6 & 10006 & ACS/WFC & $F 435 W$ & j8vp04010 & 2200 \\
\hline NB106 & & $S$ & star & 6 & 10006 & ACS/WFC & $F 435 W$ & j8vp02010 & 2200 \\
\hline AU007 & & & star & 6 & 10006 & ACS/WFC & $F 435 W$ & j8vp03010 & 2200 \\
\hline AU008 & & & globular cluster? & 2 & 10006 & ACS/WFC & $F 435 W$ & j8vp05010 & 2200 \\
\hline AU010 & & & globular cluster & 1 & 10118 & ACS/WFC & $F 814 W$ & j8zs05a6q & 507 \\
\hline M039 & & & faint & 2 & 10407 & ASC/WFC & F606W & j96q02010 & 1860 \\
\hline M047 & & & faint & 2 & 10407 & ASC/WFC & F606W & j96q04010 & 3315 \\
\hline M050 & & & faint, not star & 2 & 10407 & ASC/WFC & F606W & j96q04010 & 3315 \\
\hline M055 & & & globular cluster? & 2 & 9767 & ACS/WFC & F606W & j8o440e9q & 774 \\
\hline BH16 & & & stars? & 2 & 10006 & ACS/WFC & $F 435 W$ & j8vp05010 & 2200 \\
\hline BH18 & & & globular cluster? & 2 & 10006 & ACS/WFC & $F 435 W$ & j8vp02010 & 2200 \\
\hline BH19 & & & globular cluster? & 2 & 9392 & ACS/WFC & F606W & j8f101mtq & 1000 \\
\hline $\mathrm{BH} 25$ & & & globular cluster? & 2 & 9480 & ACS/WFC & $F 775 W$ & j8hohleeq & 700 \\
\hline
\end{tabular}

